\title{
FRAMED KNOT CONTACT HOMOLOGY
}

\author{
LENHARD NG
}

\begin{abstract}
We extend knot contact homology to a theory over the ring $\mathbb{Z}\left[\lambda^{ \pm 1}, \mu^{ \pm 1}\right]$, with the invariant given topologically and combinatorially. The improved invariant, which is defined for framed knots in $S^{3}$, can distinguish many pairs of knots, including mutants, and can also be defined for knots in arbitrary manifolds. It contains the Alexander polynomial and naturally produces a two-variable polynomial knot invariant which is related to the $A$-polynomial.
\end{abstract}

\section{INTRODUCTION}

This may be viewed as the third in a series of papers on knot contact homology, following $\mathrm{Ng} 2, \mathrm{Ng} 3$. In this paper, we extend the knot invariants of $\mathrm{Ng} 2, \mathbf{N g} 3$, which were defined over the base ring $\mathbb{Z}$, to invariants over the larger ring $\mathbb{Z}\left[\lambda^{ \pm 1}, \mu^{ \pm 1}\right]$. The new invariants, defined both for knots in $S^{3}$ or $\mathbb{R}^{3}$ and for more general knots, contain a large amount of information not contained in the original formulation of knot contact homology, which corresponds to specifying $\lambda=\mu=1$. We will recapitulate definitions from the previous papers where necessary, so that the results from this paper can hopefully be understood independently of $\mathrm{Ng} 2, \mathrm{Ng} 3$, although some proofs rely heavily on the previous papers.

The motivation for the invariant given by knot contact homology comes from symplectic geometry and in particular the Symplectic Field Theory of Eliashberg, Givental, and Hofer [EGH]. Our approach in this paper and its predecessors is to view the invariant topologically and combinatorially, without using the language of symplectic geometry. Work is currently in progress to show that the invariant defined here does actually give the "Legendrian contact homology" of a certain canonically defined object. For more details, see $[\mathrm{Ng} 2, \S 3]$ or the end of this section, where we place the current work in context within the general subject of holomorphic curves in symplectic manifolds.

We introduce the invariant in Section 2, For a knot in $\mathbb{R}^{3}$, the form for the invariant which carries the most information is a differential graded algebra over $\mathbb{Z}\left[\lambda^{ \pm 1}, \mu^{ \pm 1}\right]$, which we call the framed knot DGA, modulo an equivalence relation known in contact geometry as stable tame isomorphism, which is a special case of quasi-isomorphism. We can define this algebra in terms of a

The author is supported by an American Institute of Mathematics Five-Year Fellowship. 
braid whose closure is the desired knot; this is the version of the invariant which is directly derived from considerations in contact geometry. There is a slightly more natural definition for the framed knot DGA in terms of a diagram of the knot, but a satisfying topological interpretation for the full invariant has yet to be discovered.

We refer to the homology of the framed knot DGA, which is also a knot invariant, as framed knot contact homology. The most tractable piece of information which can be extracted from the framed knot DGA is the degree 0 piece of this homology, which we call the cord algebra of the knot because it affords a natural topological reformulation in terms of cords, in the spirit of Ng3. The cord algebra can easily be extended to knots in arbitrary manifolds using homotopy groups, specifically the peripheral information attached to the knot group. It seems likely that this general cord algebra measures the degree 0 part of the corresponding Legendrian contact homology, as for knots in $S^{3}$, but a proof would need to expand our current technology for calculating contact homology.

The framed knot DGA contains a fair amount of "classical" information about the knot. In particular, one can deduce the Alexander polynomial from a certain canonical linearization of the algebra. More interestingly, one can use the framed knot DGA, or indeed the cord algebra derived from it, to define a two-variable polynomial knot invariant which we call the augmentation polynomial. The augmentation polynomial, in turn, has a factor which is the well-studied $A$-polynomial introduced in CCGLS. In particular, a result of Dunfield and Garoufalidis DG] about the nontriviality of the $A$-polynomial implies that the cord algebra (and hence framed knot contact homology) distinguishes the unknot from all other knots. There is a close relationship between knot contact homology and $S L_{2} \mathbb{C}$ representations of the knot complement, as evidenced by the link to the $A$-polynomial, but there does not seem to be an interpretation for the framed knot DGA purely in terms of character varieties.

On a related topic, the cord algebra is a reasonably effective tool to distinguish knots. Certainly it can tell apart knots which have different Alexander polynomials, and it can also distinguish mirrors (e.g., left handed and right handed trefoils). It can even distinguish knots which have the same $A$ polynomial, such as the Kinoshita-Terasaka knot and its Conway mutant. The tool used to produce such results is a collection of numerical invariants derived from the cord algebra, called augmentation numbers, which can be readily calculated by computer. It is even remotely possible that the cord algebra could be a complete knot invariant.

Here is an outline of the paper. Section 2 contains the various definitions of the knot invariant, and the nontrivial portions of the proofs of their invariance and equivalence are given in Section 3. In Section 4. we discuss properties of the invariant, including the aforementioned relation with the Alexander polynomial. Section [5] examines some information which can 
be extracted from the invariant, including augmentation numbers and the augmentation polynomial, and discusses the relation with the $A$-polynomial.

We conclude this section with some remarks about the contact geometry of the framed knot DGA. As discussed in $\mathrm{Ng2}$, the knot DGA of a knot in $\mathbb{R}^{3}$ measures the Legendrian contact homology of a naturally defined Legendrian torus (the unit conormal to the knot) in the contact manifold $S T^{*} \mathbb{R}^{3}$. In its most general form (see [ENS]), Legendrian contact homology is defined over the group ring of the first homology group of the Legendrian submanifold: briefly, the group ring coefficients arise from considering the boundaries of the holomorphic disks used in Legendrian contact homology, which lie in the Legendrian submanifold and can be closed to closed curves by adding capping paths joining endpoints of Reeb chords.

In our case, the Legendrian torus is essentially the boundary of a tubular neighborhood of the knot, and the group ring $\mathbb{Z} H_{1}\left(T^{2}\right)$ can be identified with $\mathbb{Z}\left[\lambda^{ \pm 1}, \mu^{ \pm 1}\right]$ once we choose a framing for the knot. The resulting DGA, whose homology is the Legendrian contact homology of the Legendrian torus, should be precisely the framed knot DGA defined above. As mentioned earlier, it is expected that this will be verified soon.

Since its introduction by Eliashberg and Hofer Eli, Legendrian contact homology has been studied in many papers, including [Che, EES, ENS, Ng1. Much of this work has focused on the lowest dimensional case, that of Legendrian knots in standard contact $\mathbb{R}^{3}$ (or other contact three-manifolds). The present manuscript, along with its predecessors $\mathrm{Ng} 2$, $\mathrm{Ng} 3$, comprises one of the first instances of a reasonably nontrivial and involved computation of Legendrian contact homology in higher dimensions. Beyond its implications for knot theory, the Legendrian contact homology in this case is interesting because it has several features not previously observed in the subject. We have seen in $\mathrm{Ng} 2$ that the knot DGA over $\mathbb{Z}_{2}$ carries augmentations which give rise to distinct Poincaré polynomials; to the author's knowledge, this had never been seen before, and in fact it may never be possible for onedimensional Legendrian knots. In the present paper, we will also give the first application known to the author of Legendrian contact homology with coefficients in the group ring, as opposed to $\mathbb{Z}_{2}$.

Acknowledgments. I would like to thank Tobias Ekholm, Yasha Eliashberg, Mike Sullivan, and Ravi Vakil for useful discussions. 


\section{The InVARIANT}

We will give four interpretations of the invariant. The first, in terms of homotopy groups, generalizes the version of the cord ring given in the appendix to Ng3. The second, in terms of cords, generalizes the original definition of the cord ring in Ng3. The third, in terms of a braid representation of the knot, and the fourth, in terms of a knot diagram, extend the definition of knot contact homology and the knot DGA from $\mathrm{Ng2}$.

Throughout this section, we work with noncommutative algebras, but for the purposes of our applications, one could just as well abelianize and work in the commutative category. This is in contrast to the situation of Legendrian knots in $\mathbb{R}^{3}$, where it is sometimes advantageous to exploit noncommutativity $\mathrm{Ng} 1$. It is possible that one could use the noncommutative structure for the cord algebra or framed knot DGA to distinguish a knot from its inverse; see Section 4.1 .

2.1. Homotopy interpretation. This version of the invariant has the advantage of being defined in the most general setup.

Let $K \subset M$ be a codimension 2 submanifold of any smooth manifold, equipped with an orientation of the normal bundle to $K$ in $M$. Denote by $\nu(K)$ a tubular neighborhood of $K$ in $M$, and choose a point $x_{0}$ on the boundary $\partial(\nu(K))$. The orientation of the normal bundle determines an element $m \in \pi_{1}\left(\partial \nu K, x_{0}\right)$ given by the oriented fiber of the $S^{1}$ bundle $\partial \nu K$ over $K$. The inclusion $\iota: \partial \nu K \hookrightarrow M \backslash K$ induces a map $\iota_{*}: \pi_{1}\left(\partial \nu K, x_{0}\right) \rightarrow$ $\pi_{1}\left(M \backslash K, x_{0}\right)$.

Denote by $R=\mathbb{Z} H_{1}(\partial \nu K)$ the group ring of the homology group $H_{1}(\partial \nu K)$, with coefficients in $\mathbb{Z}$; the usual abelianization map from $\pi_{1}$ to $H_{1}$ yields a map $p: \pi_{1}\left(\partial \nu K, x_{0}\right) \rightarrow R$. Let $\mathcal{A}$ denote the tensor algebra over $R$ freely generated by the elements of the group $\pi_{1}\left(M \backslash K, x_{0}\right)$, where we view $\pi_{1}\left(M \backslash K, x_{0}\right)$ as a set. We write the image of $\gamma \in \pi_{1}\left(M \backslash K, x_{0}\right)$ in $\mathcal{A}$ as $[\gamma]$; then $\mathcal{A}$ is generated as an $R$-module by words of the form $\left[\gamma_{1}\right] \cdots\left[\gamma_{k}\right]$, $k \geq 0$. (Note that $\left[\gamma_{1} \gamma_{2}\right] \neq\left[\gamma_{1}\right]\left[\gamma_{2}\right]$ in $\mathcal{A}$.)

Definition 2.1. Let $\mathcal{I} \subset \mathcal{A}$ be the subalgebra generated by the following elements of $\mathcal{A}$ :

(1) $[e]-1-p(m)$, where $e$ is the identity in $\pi_{1}(M \backslash K)$ and 1 is the unit in $\mathcal{A}$;

(2) $\left[\gamma \iota_{*}\left(\gamma^{\prime}\right)\right]-p\left(\gamma^{\prime}\right)[\gamma]$ and $\left[\iota_{*}\left(\gamma^{\prime}\right) \gamma\right]-p\left(\gamma^{\prime}\right)[\gamma]$, for any $\gamma^{\prime} \in \pi_{1}(\partial \nu K)$ and $\gamma \in \pi_{1}(M \backslash K)$

(3) $\left[\gamma_{1} \gamma_{2}\right]+\left[\gamma_{1} \iota_{*}(m) \gamma_{2}\right]-\left[\gamma_{1}\right] \cdot\left[\gamma_{2}\right]$, for any $\gamma_{1}, \gamma_{2} \in \pi_{1}(M \backslash K)$.

The cord algebra of $K \subset M$ is the algebra $\mathcal{A} / \mathcal{I}$, over the ring $R$.

This algebra is evidently an isotopy invariant of $K$. We note that the relation $[e]=1+p(m)$ in $\mathcal{A} / \mathcal{I}$ is chosen to give consistency between the other two relations; the third relation gives

$$
\left[\gamma_{1}\right][e]=\left[\gamma_{1} e\right]+\left[\gamma_{1} \iota_{*}(m) e\right]=\left[\gamma_{1}\right]+\left[\gamma_{1} \iota_{*}(m)\right]
$$


for $\gamma_{1} \in \pi_{1}(M \backslash K)$, while the second relation gives $\left[\gamma_{1}\right]+\left[\gamma_{1} \iota_{*}(m)\right]=$ $(1+p(m))\left[\gamma_{1}\right]$.

In our case of interest, $K$ is an oriented knot in $M=S^{3}$ (or $\mathbb{R}^{3}$ ), $\partial \nu K$ is topologically a 2-torus, and $H_{1}(\partial \nu K)=\mathbb{Z}^{2}$. A framing of $K$ gives a set $\{\lambda, \mu\}$ of generators of $H_{1}(\partial \nu K)$, where $\lambda$ is the longitude of $K$ and $\mu$ is the meridian. Given a framing, we can hence identify $R=\mathbb{Z} H_{1}(\partial \nu K)$ with the $\operatorname{ring} \mathbb{Z}\left[\lambda^{ \pm 1}, \mu^{ \pm 1}\right]$.

Definition 2.2. Let $K \subset S^{3}$ be a framed knot, and let $l, m$ denote the homotopy classes of the longitude and meridian of $K$ in $\pi_{1}\left(S^{3} \backslash K\right)$. The framed cord algebra of $K$ is the tensor algebra over $\mathbb{Z}\left[\lambda^{ \pm 1}, \mu^{ \pm 1}\right]$ freely generated by $\pi_{1}\left(S^{3} \backslash K\right)$, modulo the relations

(1) $[e]=1+\mu$;

(2) $[\gamma m]=[m \gamma]=\mu[\gamma]$ and $[\gamma l]=[l \gamma]=\lambda[\gamma]$, for $\gamma \in \pi_{1}\left(S^{3} \backslash K\right)$;

(3) $\left[\gamma_{1} \gamma_{2}\right]+\left[\gamma_{1} m \gamma_{2}\right]=\left[\gamma_{1}\right]\left[\gamma_{2}\right]$, for $\gamma \in \pi_{1}\left(S^{3} \backslash K\right)$.

It is clear that, for framed knots, this definition agrees with Definition 2.1 above.

A few remarks are in order. First, the condition $[\gamma m]=[m \gamma]=\mu[\gamma]$ in (2) of Definition 2.2 is unnecessary since it follows from (11) and (3) with $\left(\gamma_{1}, \gamma_{2}\right)=(\gamma, e)$ or $(e, \gamma)$.

Second, changing the framing of the knot by $k \in \mathbb{Z}$ has the effect of replacing $\lambda$ by $\lambda \mu^{k}$ in the cord algebra. If we change $K$ to the mirror of $K$, with the corresponding framing, then the cord algebra changes by replacing $\mu$ by $\mu^{-1}$; if we reverse the orientation of $K$, then the cord algebra changes by replacing $\lambda$ by $\lambda^{-1}$ and $\mu$ by $\mu^{-1}$.

Finally, as an illustration of Definition 2.2, we compute the framed cord algebra for the 0 -framed unknot in $S^{3}$. Here $\pi_{1}\left(S^{3} \backslash K\right) \cong \mathbb{Z}$ is generated by the meridian $m$, and the relations in Definition 2.2 imply that $[e]=1+\mu$, $[m]=\mu[e]$, and $[e]=\lambda[e]$, and so the framed cord algebra is

$$
\mathbb{Z}\left[\lambda^{ \pm 1}, \mu^{ \pm 1}\right] /((\lambda-1)(\mu+1)) \text {. }
$$

2.2. Cord interpretation. The homotopy definition of the cord algebra given above is perhaps not the most useful formulation from a computational standpoint. Here we give another topological definition of the cord algebra, in terms of the paths which we termed "cords" in Ng3.

Definition 2.3. Let $K \subset S^{3}$ be an oriented knot, and let $*$ be a fixed point on $K$. A cord of $(K, *)$ is any continuous path $\gamma:[0,1] \rightarrow S^{3}$ with $\gamma^{-1}(K)=\{0,1\}$ and $\gamma^{-1}(\{*\})=\emptyset$. Let $\mathcal{A}_{K}$ denote the tensor algebra over $\mathbb{Z}\left[\lambda^{ \pm 1}, \mu^{ \pm 1}\right]$ freely generated by the set of homotopy classes of cords of $(K, *)$.

Note that this definition differs slightly from the definition of cords in $\mathrm{Ng} 3$. As in Ng3, we distinguish between a knot and its cords in diagrams by drawing the knot more thickly. 
In $\mathcal{A}_{K}$, we define skein relations as follows:
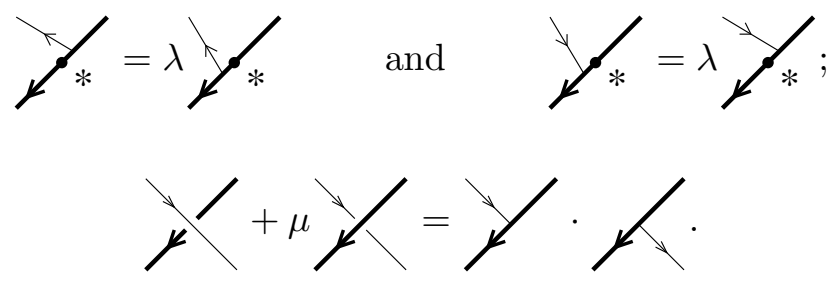

These diagrams are understood to depict some neighborhood in $S^{3}$ outside of which the diagrams agree. To clarify the diagrams, if one pushes either of the cords on the left hand side of (3) to intersect the knot, then the cord splits into the two cords on the right hand side of (3); also, the cord in (11) is any contractible cord. Note that the skein relations are considered as diagrams in space rather than in the plane. For example, (3) is equivalent to

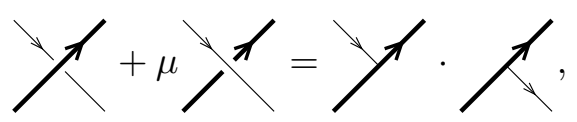

even though the two relations are not the same when interpreted as plane diagrams.

Definition 2.4. The cord algebra of $(K, *)$ is $\mathcal{A}_{K}$ modulo the relations (11), (2)) and (3).

Note that the definition of the cord algebra of $(K, *)$, if we set $\lambda=\mu=1$, is the same as the definition of the cord ring of $K$ from $\mathrm{Ng} 3$, after we change variables by replacing every cord by -1 times itself.

Proposition 2.5. The cord algebra of $(K, *)$ is independent, up to isomorphism, of the choice of the point $*$, and is isomorphic to the framed cord algebra of Definition 2.2 when $K \subset S^{3}$ is given the 0 framing.

Proof. To see the isomorphism between the algebras specified by Definitions 2.2 and 2.4 connect the base point $x_{0} \in \partial \nu K$ from the homotopy interpretation to a nearby point $x \in K$ (with $x \neq *$ ) via some path. By conjugating by this path, we can associate, to any loop $\gamma$ representing an element of $\pi_{1}\left(S^{3} \backslash K, x_{0}\right)$, a cord $\tilde{\gamma}$ beginning and ending at $x$, and homotopic loops are mapped to homotopic cords. The map from the framed cord algebra with framing 0 to the cord algebra of Definition [2.4 sends $[\gamma]$ to $\mu^{\operatorname{lk}(\gamma, K)}[\tilde{\gamma}]$, where $\mathrm{lk}$ is the linking number. It is easily verified that this map is an isomorphism. Note that the 0 framing is necessary so that a cord following the longitude of $K$ is identified with $\lambda$ times the contractible cord. 
It follows readily from Definition 2.4 that the cord algebra of a knot $K$ is finitely generated and finitely presented. Any cord, in fact, can be expressed in terms of the so-called minimal binormal chords of the knot, which are the local length minimizers among the line segments beginning and ending on the knot (imagine a general cord as a rubber band and pull it tight). For a description of how we can calculate the cord algebra from a diagram of the knot, see Section 2.4 .

2.3. Differential graded algebra interpretation I. This version of the invariant is the original one, derived from holomorphic curve theory and contact homology. The invariant we define here is actually "better" than the cord algebra defined above; it is a differential graded algebra whose homology in degree 0 is the cord algebra. It is an open question to develop a topological interpretation for the full differential graded algebra, using cords or homotopy groups as in Sections 2.1 and 2.2

We recall some definitions from $\mathrm{Ng} 2$, slightly modified for our purposes. Let $B_{n}$ be the braid group on $n$ strands, and let $\mathcal{A}_{n}$ denote the tensor algebra over $\mathbb{Z}\left[\lambda^{ \pm 1}, \mu^{ \pm 1}\right]$ freely generated by the $n(n-1)$ generators $a_{i j}$, with $1 \leq i, j \leq n$ and $i \neq j$. There is a representation $\phi: B_{n} \rightarrow \operatorname{Aut} \mathcal{A}_{n}$ defined on the usual generators $\sigma_{1}, \ldots, \sigma_{n-1}$ of $B_{n}$ as follows:

$$
\phi_{\sigma_{k}}:\left\{\begin{array}{cll}
a_{k i} & \mapsto-a_{k+1, i}-a_{k+1, k} a_{k i} & i \neq k, k+1 \\
a_{i k} & \mapsto-a_{i, k+1}-a_{i k} a_{k, k+1} & i \neq k, k+1 \\
a_{k+1, i} & \mapsto a_{k i} & i \neq k, k+1 \\
a_{i, k+1} & \mapsto a_{i k} & i \neq k, k+1 \\
a_{k, k+1} & \mapsto a_{k+1, k} & \\
a_{k+1, k} & \mapsto a_{k, k+1} & i, j \neq k, k+1 . \\
a_{i j} & \mapsto a_{i j}
\end{array}\right.
$$

Write $\phi_{B}$ as the image of a braid $B \in B_{n}$ under $\phi$. There is a similar representation $\phi^{\text {ext }}: B_{n} \rightarrow$ Aut $\mathcal{A}_{n+1}$ given by the composition of the inclusion $B_{n} \hookrightarrow B_{n+1}$ with the map $\phi$ on $B_{n+1}$, where the inclusion adds a strand we label $*$ to the $n$-strand braid. For $B \in B_{n}$, we can then define $n \times n$ matrices $\Phi_{B}^{L}, \Phi_{B}^{R}$ with coefficients in $\mathcal{A}_{n}$ by the defining relations

$$
\phi_{B}^{\text {ext }}\left(a_{i *}\right)=\sum_{j=1}^{n}\left(\Phi_{B}^{L}\right)_{i j} a_{j *} \quad \text { and } \quad \phi_{B}^{\text {ext }}\left(a_{* j}\right)=\sum_{i=1}^{n} a_{* i}\left(\Phi_{B}^{R}\right)_{i j} .
$$

Fix a braid $B \in B_{n}$. Let $\mathcal{A}$ be the graded tensor algebra over $\mathbb{Z}\left[\lambda^{ \pm 1}, \mu^{ \pm 1}\right]$ generated by: $a_{i j}, 1 \leq i, j \leq n, i \neq j$, of degree $0 ; b_{i j}$ and $c_{i j}, 1 \leq i, j \leq n$, of degree $1 ;$ and $d_{i j}, 1 \leq i, j \leq n$, and $e_{i}, 1 \leq i \leq n$, of degree 2. Assemble the $a, b, c, d$ generators of $\mathcal{A}$ into $n \times n$ matrices as follows: $B=\left(b_{i j}\right), C=\left(c_{i j}\right)$, 
$D=\left(d_{i j}\right)$, and $A=\left(\tilde{a}_{i j}\right)$, where

$$
\tilde{a}_{i j}= \begin{cases}\mu a_{i j}, & i<j \\ a_{i j}, & i>j \\ -1-\mu, & i=j .\end{cases}
$$

Also define $\Lambda$ to be the $n \times n$ diagonal matrix $\operatorname{diag}(\lambda, 1, \ldots, 1)$.

Definition 2.6. Let $B \in B_{n}$, and let $\mathcal{A}$ be the algebra given above. Define a differential $\partial$ on the generators of $\mathcal{A}$ by

$$
\begin{aligned}
\partial A & =0 \\
\partial B & =\left(1-\Lambda \cdot \Phi_{B}^{L}\right) \cdot A \\
\partial C & =A \cdot\left(1-\Phi_{B}^{R} \cdot \Lambda^{-1}\right) \\
\partial D & =B \cdot\left(1-\Phi_{B}^{R} \cdot \Lambda^{-1}\right)-\left(1-\Lambda \cdot \Phi_{B}^{L}\right) \cdot C \\
\partial e_{i} & =\left(B+\Lambda \cdot \Phi_{B}^{L}(A) \cdot C\right)_{i i},
\end{aligned}
$$

where $\cdot$ denotes matrix multiplication, and extend $\partial$ to $\mathcal{A}$ via the Leibniz rule. Then $(\mathcal{A}, \partial)$ is the framed knot $D G A$ of $B$.

Here "DGA" is the abbreviation commonly used in the subject for a (semifree) differential graded algebra. We remark that if we set $\lambda=\mu=1$, we recover the definition of the knot DGA over $\mathbb{Z}$ from $\mathrm{Ng} 2$.

There is a standard notion of equivalence between DGAs known as stable tame isomorphism, originally due to Chekanov [Che] we now briefly review its definition, in the version which we need. Note that an important property of stable tame isomorphism is that it preserves homology (see [ENS]).

Suppose that we have two DGAs $(\mathcal{A}, \partial)$ and $\left(\mathcal{A}^{\prime}, \partial^{\prime}\right)$, where $\mathcal{A}, \mathcal{A}^{\prime}$ are tensor algebras over $\mathbb{Z}\left[\lambda^{ \pm 1}, \mu^{ \pm 1}\right]$ generated by $a_{1}, \ldots, a_{n}$ and $a_{1}^{\prime}, \ldots, a_{n}^{\prime}$, respectively. An algebra map $\psi: \mathcal{A} \rightarrow \mathcal{A}^{\prime}$ is an elementary isomorphism if it is a graded chain map and, for some $i$,

$$
\psi\left(a_{j}\right)= \begin{cases}\alpha a_{i}^{\prime}+v, & j=i, \\ a_{j}^{\prime}, & j \neq i,\end{cases}
$$

where $\alpha$ is a unit in $\mathbb{Z}\left[\lambda^{ \pm 1}, \mu^{ \pm 1}\right]$ and $v$ is in the subalgebra of $\mathcal{A}^{\prime}$ generated by $a_{1}^{\prime}, \ldots, a_{i-1}^{\prime}, a_{i+1}^{\prime}, \ldots, a_{n}^{\prime}$. A tame isomorphism between DGAs is a composition of elementary isomorphisms. Let $\left(\mathcal{E}^{i}, \partial^{i}\right)$ be the tensor algebra on two generators $e_{1}^{i}, e_{2}^{i}$, with $\operatorname{deg} e_{1}^{i}-1=\operatorname{deg} e_{2}^{i}=i$ and differential induced by $\partial^{i} e_{1}^{i}=e_{2}^{i}, \partial^{i} e_{2}^{i}=0$. The degree- $i$ algebraic stabilization of a $\operatorname{DGA}(\mathcal{A}, \partial)$ is the coproduct of $\mathcal{A}$ with $\mathcal{E}^{i}$, with the differential induced from $\partial$ and $\partial^{i}$. Finally, two DGAs are stable tame isomorphic if they are tamely isomorphic after some (possibly trivial, possibly different) number of algebraic stabilizations of each.

We can now state the invariance result for framed knot DGAs. For any braid $B \in B_{n}$, define the writhe $w(B)$, as usual, to be the sum of the exponents of the word in $\sigma_{1}, \ldots, \sigma_{n-1}$ comprising $B$. 
Theorem 2.7. If braids $B$ and $B^{\prime}$ have isotopic knot closures, then the framed knot DGA for $B^{\prime}$ is stable tame isomorphic to the DGA obtained by replacing $\lambda$ by $\lambda \mu^{w\left(B^{\prime}\right)-w(B)}$ in the framed knot $D G A$ for $B$.

We defer the proof of Theorem 2.7 to Section 3.1

Definition 2.8. Let $K \subset S^{3}$ be a knot. The $f$-framed knot DGA of $K$ is the (stable tame isomorphism class of the) framed knot DGA of any braid $B$ whose closure is $K$ and which has writhe $f$. We will often refer to the 0 -framed knot DGA, which can be obtained from the $f$-framed knot DGA by replacing $\lambda$ by $\lambda \mu^{f}$, as the framed knot $D G A$.

Since mirroring affects the framed knot DGA nontrivially, Definition 2.8 is not completely clear until we specify what positive and negative braid crossings look like when the braid is embedded in $\mathbb{R}^{3}$. We choose the convention that a positive crossing in a braid (i.e., a generator $\sigma_{k}$ ) is one in which the strands rotate around each other counterclockwise. Thus the writhe of a braid is negative the writhe of the knot which is its closure; we will henceforth only use the term "writhe" vis-à-vis braids.

Since stable tame isomorphic DGAs have isomorphic homology, the homology of the framed knot DGA produces another framed knot invariant.

Definition 2.9. Let $K$ be a framed knot in $S^{3}$ with framing $f$, or a knot with framing 0 if no framing is specified. The framed knot contact homology of $K$, denoted $H C_{*}(K)$, is the graded homology of the framed knot DGA of $K$.

Note that $H C_{*}(K)$ only exists in dimensions $* \geq 0$. The following result connects the invariant defined in this section to the invariants from the two previous sections.

Theorem 2.10. Let $K \subset S^{3}$ be a knot. Then the degree 0 framed knot contact homology $H C_{0}(K)$, viewed as an algebra over $\mathbb{Z}\left[\lambda^{ \pm 1}, \mu^{ \pm 1}\right]$, is isomorphic to the cord algebra of $K$.

We will indicate a proof of Theorem 2.10 in Section 3.2

Corollary 2.11 (cf. Ng2, Prop. 4.2]). If $B \in B_{n}$ is a braid which has closure $K$, then the cord algebra of $K$ is the result of replacing $\lambda$ by $\lambda \mu^{-w(B)}$ in $\mathcal{A}_{n} / \mathcal{I}$, where $\mathcal{I}$ is the subalgebra generated by the entries of the matrices $\left(1-\Lambda \cdot \Phi_{B}^{L}\right) \cdot A$ and $A \cdot\left(1-\Phi_{B}^{R} \cdot \Lambda^{-1}\right)$.

Potentially the framed knot DGA of a knot contains much more information than the cord algebra. However, it seems difficult to compute $H C_{*}$ for $*>0$, and it remains an open question whether stable tame isomorphism is a stronger relation than quasi-isomorphism (i.e., having isomorphic homology).

As an example, we compute the cord algebra for the left handed trefoil, which is the closure of the braid $\sigma_{1}^{3} \in B_{2}$. As calculated in $\mathrm{Ng} 2$, we have

$$
\Phi_{\sigma_{1}^{3}}^{L}=\left(\begin{array}{cc}
2 a_{21}-a_{21} a_{12} a_{21} & 1-a_{21} a_{12} \\
-1+a_{12} a_{21} & a_{12}
\end{array}\right) \quad \text { and } \quad \Phi_{\sigma_{1}^{3}}^{R}=\left(\begin{array}{cc}
2 a_{12}-a_{12} a_{21} a_{12} & -1+a_{12} a_{21} \\
1-a_{21} a_{12} & a_{21}
\end{array}\right)
$$




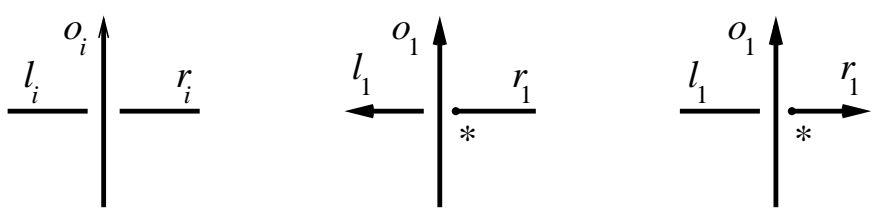

FiguRE 1. Labeling the strands at a knot crossing. The left diagram is crossing $i$ for $i \geq 2$; the center and right diagrams are crossing 1 for $\epsilon_{1}=1$ and $\epsilon_{1}=-1$, respectively.

and hence $H C_{0}$ is $\mathcal{A}_{2}$ modulo the entries of the matrices

$$
\left(1-\Lambda \cdot \Phi_{\sigma_{1}^{3}}^{L}\right) \cdot A=\left(\begin{array}{cc}
-1-\mu+2 \lambda a_{21}+\lambda \mu a_{21}-\lambda a_{21} a_{12} a_{21} & \lambda+\lambda \mu+a_{12}-(3 \lambda+\lambda \mu) a_{21} a_{12}+\lambda a_{21} a_{12} a_{21} a_{12} \\
-1-\mu+\mu a_{21}+a_{12} a_{21} & -1-\mu+2 a_{12}+\mu a_{12}-a_{12} a_{21} a_{12}
\end{array}\right)
$$

and

$$
A \cdot\left(1-\Phi_{\sigma_{1}^{3}}^{R} \cdot \Lambda^{-1}\right)=\left(\begin{array}{cc}
\lambda^{-1}\left(-\lambda-\lambda \mu+(1+2 \mu) a_{12}-\mu a_{12} a_{21} a_{12}\right) & -1-\mu+a_{12}+\mu a_{12} a_{21} \\
\lambda^{-1}\left(1+\mu+\lambda \mu a_{21}-(1+3 \mu) a_{21} a_{12}+\mu a_{21} a_{12} a_{21} a_{12}\right) & -1-\mu+a_{21}+2 \mu a_{21}-\mu a_{21} a_{12} a_{21}
\end{array}\right)
$$

once we replace $\lambda$ by $\lambda \mu^{-3}$. As in the computation from $\mathrm{Ng} 2$, it follows that in $H C_{0}$, we have $a_{21}=\mu^{2} a_{12}-\mu^{2}+1$, and, setting $a_{12}=x$, we find that

$H C_{0}(\mathrm{LH}$ trefoil $) \cong\left(\mathbb{Z}\left[\lambda^{ \pm 1}, \mu^{ \pm 1}\right]\right)[x] /\left((x-1)\left(\mu^{2} x+\mu+1\right), \lambda \mu^{2} x-\mu^{3} x-\lambda \mu^{2}+\lambda\right)$.

We can then deduce $H C_{0}$ for the right handed trefoil by replacing $\mu$ by $\mu^{-1}$ :

$H C_{0}(\mathrm{RH}$ trefoil $) \cong\left(\mathbb{Z}\left[\lambda^{ \pm 1}, \mu^{ \pm 1}\right]\right)[x] /\left((x-1)\left(x+\mu^{2}+\mu\right), \lambda \mu x-x+\lambda \mu^{3}-\lambda \mu\right)$.

2.4. Differential graded algebra interpretation II. The definition of the framed knot DGA from Section 2.3 is somewhat formal and inscrutable. Here we give another formulation for the DGA, using a diagram of the knot rather than a braid presentation. This is based on the cord formulation for the invariant, and improves on Section 4.4 of $\mathrm{Ng} 3$.

We first describe how to use a knot diagram to calculate the cord algebra. Suppose that we have a knot diagram of $K$ with $n$ crossings; see Figure 1 The crossings divide the diagram of the knot into $n$ connected components, which we label $1, \ldots, n$; also number the crossings $1, \ldots, n$ in some way. Crossing $\alpha$ can be represented as $\left(o_{\alpha}, l_{\alpha}, r_{\alpha}\right)$, where $o_{\alpha}$ is the overcrossing strand, and $l_{\alpha}$ and $r_{\alpha}$ are the left and right undercrossing strands from the point of view of the orientation on $o_{\alpha}$. Choose the point $*$ of Definition 2.4 to be the undercrossing point of crossing 1 , and define $\epsilon_{1}$ to be 1 if crossing 1 is a positive crossing, and -1 if it is negative.

Let $\mathcal{A}_{n}$ denote the tensor algebra over $\mathbb{Z}\left[\lambda^{ \pm 1}, \mu^{ \pm 1}\right]$ generated by $a_{i j}$, where $1 \leq i, j \leq n$ and $i \neq j$, and set $a_{i i}=1+\mu$ for $1 \leq i \leq n$. Define $\mathcal{I}_{K}^{\text {diagram }} \subset \mathcal{A}_{n}$ to be the subalgebra generated by the following:

$$
\begin{array}{ll}
a_{j l_{i}}+\mu a_{j r_{i}}-a_{j o_{i}} a_{o_{i} l_{i}}, \mu a_{l_{i} j}+a_{r_{i} j}-a_{l_{i} o_{i}} a_{o_{i} j}, & 2 \leq i \leq n \text { and } 1 \leq j \leq n \\
a_{j l_{1}}+\lambda^{\epsilon 1} \mu a_{j r_{1}}-a_{j o_{1}} a_{o_{1} l_{1}}, \mu a_{l_{1} j}+\lambda^{-\epsilon_{1}} a_{r_{1} j}-a_{l_{1} o_{1}} a_{o_{1} j}, & 1 \leq j \leq n
\end{array}
$$




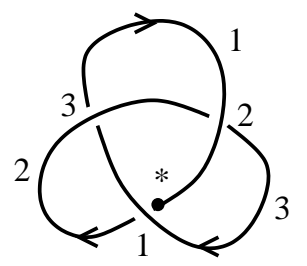

FiguRE 2. A diagram of the right hand trefoil, with components and crossings labeled.

This subalgebra is constructed to contain all of the relations imposed by (3) of Definition [2.4, see also Ng3, §4.4].

Proposition 2.12. The cord algebra of $(K, *)$ is given by $\mathcal{A}_{n} / \mathcal{I}_{K}^{\text {diagram }}$.

Proof. Same as the proof of Proposition 4.8 in Ng3.

We can express the relations defining $\mathcal{I}_{K}^{\text {diagram }}$ more neatly in terms of matrices. Write $X_{i, j}$ for the $n \times n$ matrix which has 1 in the $i j$ entry and 0 everywhere else. Let $\Psi^{L}, \Psi^{R}, \Psi_{1}^{L}, \Psi_{1}^{R}, \Psi_{2}^{L}, \Psi_{2}^{R}$ be the $n \times n$ matrices given as follows:

$$
\begin{array}{ll}
\Psi_{L}^{1}=\lambda^{-\epsilon_{1}} X_{1, r_{1}}+\sum_{\alpha \neq 1} X_{\alpha, r_{\alpha}} & \Psi_{R}^{1}=\lambda^{\epsilon_{1}} \mu X_{r_{1}, 1}+\mu \sum_{\alpha \neq 1} X_{r_{\alpha}, \alpha} \\
\Psi_{L}^{2}=\sum_{\alpha}\left(\mu X_{\alpha, l_{\alpha}}-a_{l_{\alpha} o_{\alpha}} X_{\alpha, o_{\alpha}}\right) & \Psi_{R}^{2}=\sum_{\alpha}\left(X_{l_{\alpha}, \alpha}-a_{o_{\alpha} l_{\alpha}} X_{o_{\alpha}, \alpha}\right) \\
\Psi_{L}=\Psi_{L}^{1}+\Psi_{L}^{2} & \Psi_{R}=\Psi_{R}^{1}+\Psi_{R}^{2} .
\end{array}
$$

This definition may seem a bit daunting, but notice that the matrices $\Psi$ are quite sparse; for example, $\Psi^{L}$ (resp. $\Psi^{R}$ ) has at most three nonempty entries in each row (resp. column).

Also, assemble the generators of $\mathcal{A}_{n}$ into a matrix $\hat{A}$ with $\hat{A}_{i i}=1+\mu$ and $\hat{A}_{i j}=a_{i j}$ for $i \neq j$. (Note that this is slightly different than the matrix $A$ from Section [2.3.) Then the generators of $\mathcal{I}_{K}^{\text {diagram }}$ are the entries of the matrices

$$
\Psi^{L} \cdot \hat{A}, \quad \hat{A} \cdot \Psi^{R} .
$$

For example, for the right handed trefoil shown in Figure 2, we have $\Psi^{L}=\left(\begin{array}{ccc}\lambda^{-1} & \mu & -a_{23} \\ -a_{31} & 1 & \mu \\ \mu & -a_{12} & 1\end{array}\right)$ and $\Psi^{R}=\left(\begin{array}{ccc}\lambda \mu & -a_{13} & 1 \\ 1 & \mu & -a_{21} \\ -a_{32} & 1 & \mu\end{array}\right)$. If we set the entries of $\Psi^{L} \cdot \hat{A}$ and $\hat{A} \cdot \Psi^{R}$ equal to 0 , we find that $a_{23}=a_{21}, a_{32}=a_{12}, a_{13}=a_{12}$, $a_{31}=a_{21}, \lambda a_{31}=a_{32}, \lambda^{-1} a_{13}=a_{23}$. Setting $a_{12}=x$, we can use the relations to compute that the cord ring of the right hand trefoil is

$$
\left(\mathbb{Z}\left[\lambda^{ \pm 1}, \mu^{ \pm 1}\right]\right)[x] /\left(x^{2}-x-\lambda \mu^{2}-\lambda \mu, x^{2}-\lambda \mu x-\lambda-\lambda \mu\right) .
$$

This agrees with the expression given in Section 2.3. after we change variables $x \rightarrow-\mu^{-1} x$. 
We now construct a differential graded algebra whose degree 0 homology is the cord algebra. Similarly to Section 2.3. the algebra is the graded tensor algebra over $\mathbb{Z}\left[\lambda^{ \pm 1}, \mu^{ \pm 1}\right]$ generated by: $a_{i j}, 1 \leq i \neq j \leq n$, of degree $0 ; b_{\alpha i}$ and $c_{i \alpha}, 1 \leq \alpha, i \leq n$, of degree 1 ; and $d_{\alpha \beta}, 1 \leq \alpha, \beta \leq n$, and $e_{\alpha}, 1 \leq \alpha \leq n$, of degree 2 . The differential is given by:

$$
\begin{aligned}
& \partial B=\Psi^{L} \cdot \hat{A} \\
& \partial C=\hat{A} \cdot \Psi^{R} \\
& \partial D=B \cdot \Psi^{R}-\Psi^{L} \cdot C \\
& \partial e_{i}=\left(B \cdot \Psi_{1}^{R}-\Psi_{2}^{L} \cdot C\right)_{i i} .
\end{aligned}
$$

Proposition 2.13. This defines a differential, and the resulting differential graded algebra is stable tame isomorphic to the framed knot DGA.

The author's current proof of Proposition 2.13 is quite laborious, involving verification of invariance under Reidemeister moves and then identification with the framed knot DGA for a particular knot diagram. Since we have no applications yet of Proposition 2.13, we omit its proof. We have nevertheless included this formulation of the framed knot DGA in hopes that it might shed light on the topology behind the DGA invariant.

We remark that, using the above expression for the cord algebra in terms of a knot diagram, we can write the cord algebra for a knot of bridge number $k$ as a quotient of $\mathcal{A}_{k}$. Express the knot as a $2 k$-plat, with $k$ vertical tangents to the knot diagram on the left and $k$ on the right, and label the $k$ leftmost strands of the diagram $1, \ldots, k$; then if we progressively construct the knot starting with these $k$ strands and moving from left to right, we can express any generator $a_{i j}$ involving a strand not among $1, \ldots, k$ in terms of the generators of $\mathcal{A}_{k}$. More generally, we can use stable tame isomorphism to express the framed knot DGA as a DGA all of whose generators have indices less than or equal to $k$, although this is more difficult to establish.

\section{Equivalence and Invariance Proofs}

Here we prove the main theorems from Section 2, invariance of the framed knot DGA (Theorem 2.7) and equivalence of the cord algebra and $H C_{0}$ (Theorem 2.10). The proofs rely heavily on the corresponding proofs in Ng2, Ng3, and the casual reader will probably want to skip to the next section.

3.1. Proof of Theorem 2.7. Theorem 2.7 is proven by following the proof of Theorem 2.10 in $\mathrm{Ng} 2$, which is the special case of Theorem 2.7 when $\lambda=\mu=1$, and making modifications where necessary. We will provide the proof at the end of this section. 
We first need an auxiliary result, which will also be useful in Section 4.1 In the definition of the framed knot DGA, we used a matrix $\Lambda=\operatorname{diag}(\lambda, 1, \ldots, 1)$. We could just as well have used a diagonal matrix where $\lambda$ is the $m$-th diagonal entry rather than the first. The result states that this does not change the framed knot DGA, up to tame isomorphism. Note that the corresponding result for $H C_{0}$ essentially amounts to the statement that the cord algebra of Definition 2.4 is independent of the base point $*$.

For any vector $v$ of length $n$, let $\Delta(v)$ be the $n \times n$ diagonal matrix whose diagonal entries are the entries of $v$. If $n$ is fixed, then for $1 \leq m \leq n$, define $v_{m}$ to be the vector $(1, \ldots, 1, \lambda, 1, \ldots, 1)$ of length $n$ with $\lambda$ as its $m$-th entry. Write $\Lambda_{m}=\Delta\left(v_{m}\right)$.

Proposition 3.1. For $B \in B_{n}$, let $(\mathcal{A}, \partial)$ be the framed knot DGA of $B$, as given in Definition 2.6. Let $(\tilde{\mathcal{A}}, \tilde{\partial})$ be the DGA obtained from the same definition, but with $\Lambda$ replaced by $\Lambda_{m}$ for some $m \leq n$. Then $(\mathcal{A}, \partial)$ and $(\tilde{\mathcal{A}}, \tilde{\partial})$ are tamely isomorphic.

Before we prove Proposition 3.1, we establish a simple lemma. Fix a braid $B \in B_{n}$ which closes to a knot. Let $s(B)$ denote the image of $B$ under the usual homomorphism $s: B_{n} \rightarrow S_{n}$ to the symmetric group; there is an obvious action of $s(B)$ on vectors of length $n$, by permuting entries.

The matrices $\Phi_{B}^{L}, \Phi_{B}^{R}$ have entries which are (noncommutative) polynomials in the $a_{i j}$ variables; for clarity, we can denote them by $\Phi_{B}^{L}(A), \Phi_{B}^{R}(A)$, where $A$ is the usual matrix of the $a_{i j}$ 's. If $\tilde{A}$ denotes the same matrix but with each $a_{i j}$ replaced by $\tilde{a}_{i j}$, then we write $\Phi_{B}^{L}(\tilde{A}), \Phi_{B}^{R}(\tilde{A})$ to be the matrices $\Phi_{B}^{L}(A), \Phi_{B}^{R}(A)$ with each $a_{i j}$ replaced by $\tilde{a}_{i j}$.

Lemma 3.2. Let $v$ be a vector of length $n$, and define $\tilde{a}_{i j}$ by the matrix equation $\tilde{A}=\Delta(v) \cdot A \cdot \Delta(v)^{-1}$; that is, $\tilde{a}_{i j}=\frac{v_{i}}{v_{j}} a_{i j}$. Then

$\Phi_{B}^{L}(\tilde{A})=\Delta(s(B) v) \cdot \Phi_{B}^{L}(A) \cdot \Delta(v)^{-1} \quad$ and $\quad \Phi_{B}^{R}(\tilde{A})=\Delta(v) \cdot \Phi_{B}^{R}(A) \cdot \Delta(s(B) v)^{-1}$.

Proof. The $i j$ entry of $\Phi_{B}^{L}(A)$ is a linear combination of words of the form $a_{s(B)(i), i_{1}} a_{i_{1} i_{2}} \cdots a_{i_{k-1} i_{k}} a_{i_{k} j}$, while the $i j$ entry of $\Phi_{B}^{L}(\tilde{A})$ is the same linear combination of words $\tilde{a}_{s(B)(i), i_{1}} \tilde{a}_{i_{1} i_{2}} \cdots \tilde{a}_{i_{k-1} i_{k}} \tilde{a}_{i_{k} j}=\frac{v_{s(B)(i)}}{v_{j}} a_{s(B)(i), i_{1}} a_{i_{1} i_{2}} \cdots a_{i_{k-1} i_{k}} a_{i_{k} j}$. Hence the $i j$ entry of $\Phi_{B}^{L}(\tilde{A})$ is $\frac{v_{s(B)(i)}}{v_{j}}$ times the $i j$ entry of $\Phi_{B}^{L}(A)$. The result follows for $\Phi_{B}^{L}$; an analogous computation yields the result for $\Phi_{B}^{R}$.

Proof of Proposition [3.1. Since the braid $B$ closes to a knot, the permutation $s(B)$ can be written as a cycle $\left(1, m_{1}, \ldots, m_{n-1}\right)$. Define a vector $v_{m}$ of length $n$ whose $i$-th entry is $\lambda$ if $i$ appears after 1 but not after $m$ in the cycle for $s(B)$; for example, for $B=\sigma_{1} \sigma_{3} \sigma_{2}^{-3} \in B_{4}$, we have $s(B)=(1243)$, and thus $v_{2}=(1, \lambda, 1,1), v_{3}=(1, \lambda, \lambda, \lambda), v_{4}=(1, \lambda, \lambda, 1)$.

To distinguish between the algebras $\mathcal{A}$ and $\tilde{\mathcal{A}}$, denote the generators of $\tilde{\mathcal{A}}$ by $\tilde{a}_{i j}, \tilde{b}_{i j}, \tilde{c}_{i j}, \tilde{d}_{i j}, \tilde{e}_{i}$; the first four families can be assembled into matrices $\tilde{A}, \tilde{B}, \tilde{C}, \tilde{D}$. We claim that the identification $\tilde{A}=\Delta\left(v_{m}\right) \cdot A \cdot \Delta\left(v_{m}\right)^{-1}$, 
$\tilde{B}=\Delta\left(v_{m}\right) \cdot B \cdot \Delta\left(v_{m}\right)^{-1}, \tilde{C}=\Delta\left(v_{m}\right) \cdot C \cdot \Delta\left(v_{m}\right)^{-1}, \tilde{D}=\Delta\left(v_{m}\right) \cdot D \cdot \Delta\left(v_{m}\right)^{-1}$, $\tilde{e}_{i}=e_{i}$ also identifies $\tilde{\partial}$ with $\tilde{\partial}$.

Indeed, under this identification, we have

$$
\begin{aligned}
\tilde{\partial} \tilde{B} & =\tilde{A}-\Lambda_{m} \cdot \Phi_{B}^{L}(\tilde{A}) \cdot \tilde{A} \\
& =\Delta\left(v_{m}\right) \cdot A \cdot \Delta\left(v_{m}\right)^{-1}-\Lambda_{m} \cdot \Delta\left(s(B) v_{m}\right) \cdot \Phi_{B}^{L}(A) \cdot A \cdot \Delta\left(v_{m}\right)^{-1} \\
& =\Delta\left(v_{m}\right) \cdot\left(A-\Lambda \cdot \Phi_{B}^{L}(A) \cdot A\right) \cdot \Delta\left(v_{m}\right)^{-1} \\
& =\partial \tilde{B}
\end{aligned}
$$

where the second equality follows from 3.2 and the third follows from the fact that $\Lambda_{m}=\Delta\left(v_{m}\right) \cdot \Lambda \cdot \Delta\left(s(B) v_{m}\right)$, by the construction of $v_{m}$. We similarly find that $\tilde{\partial}=\partial$ on $\tilde{C}, \tilde{D}$, and $\tilde{e}_{i}$. Thus the identification gives a (tame) isomorphism between $\mathcal{A}$ and $\tilde{\mathcal{A}}$ which intertwines $\partial$ and $\tilde{\partial}$, as desired.

Sketch of proof of Theorem 2.7. The theorem can be proved in a way precisely following the proof of Theorem 2.10 in $\mathrm{Ng} 2$, making alterations where necessary. It suffices to establish the theorem when $B$ and $B^{\prime}$ are related by one of the Markov moves. We consider each in turn, giving the identification of generators which establishes the desired stable tame isomorphism, and leaving the easy but extremely tedious verifications to the reader.

Conjugation. Here $\tilde{B}=\sigma_{k}^{-1} B \sigma_{k}$ for some $k$. It is most convenient to prove that a slightly different form for the framed knot DGA is the same for $B$ and $\tilde{B}$. More precisely, it is easy to check that the framed knot DGA for any braid $B$ is stable tame isomorphic to the "modified framed knot DGA" with generators $\left\{a_{i j} \mid 1 \leq i, j \leq n, i \neq j\right\}$ of degree $0,\left\{b_{i j} \mid 1 \leq i, j \leq n, i \neq j\right\}$ and $\left\{c_{i j}, d_{i j} \mid 1 \leq i, j \leq n\right\}$ of degree 1 , and $\left\{e_{i j}, f_{i j} \mid 1 \leq i, j \leq n\right\}$ of degree 2 , and differential

$$
\begin{aligned}
\partial A & =0 \\
\partial B & =A-\Lambda \cdot \phi_{B}(A) \cdot \Lambda^{-1} \\
\partial C & =\left(1-\Lambda \cdot \Phi_{B}^{L}(A)\right) \cdot A \\
\partial D & =A \cdot\left(1-\Phi_{B}^{R}(A) \cdot \Lambda^{-1}\right) \\
\partial E & =B-D-C \cdot \Phi_{B}^{R}(A) \cdot \Lambda^{-1} \\
\partial F & =B-C-\Lambda \cdot \Phi_{B}^{L}(A) \cdot D,
\end{aligned}
$$

where we set $b_{i i}=0$ for all $i$.

Define $\tilde{\Lambda}$ to be $\Lambda$ if $k \geq 2$, and $\Lambda_{2}$ if $k=1$. By Proposition 3.1. the modified framed knot DGA for $\tilde{B}$ is tamely isomorphic to the same DGA but with $\Lambda$ replaced by $\tilde{\Lambda}$; call the latter $\operatorname{DGA}(\tilde{\mathcal{A}}, \tilde{\partial})$, with generators $\tilde{a}_{i j}, \tilde{b}_{i j}, \tilde{c}_{i j}, \tilde{d}_{i j}, \tilde{e}_{i}$. It suffices to exhibit a tame isomorphism between $(\tilde{\mathcal{A}}, \tilde{\partial})$ and the modified framed knot DGA $(\mathcal{A}, \partial)$ of $B$.

Let $\epsilon_{k}$ be $\lambda$ if $k=1$ and 1 otherwise, and, for $i \neq j$, let $a_{i j}^{\prime}$ be the $i j$ entry in $A$, i.e., $a_{i j}^{\prime}=a_{i j}$ if $i>j$ and $a_{i j}^{\prime}=\mu a_{i j}$ if $i<j$. Now identify generators 
of $\mathcal{A}$ and $\tilde{\mathcal{A}}$ as follows: $\tilde{a}_{i j}=\phi_{B}\left(a_{i j}\right)$;

$$
\begin{array}{rlrl}
\tilde{b}_{k i}=-b_{k+1, i}-\epsilon_{k}^{-1} \phi\left(a_{k+1, k}\right) b_{k i}-b_{k+1, k} a_{k i}^{\prime} & i \neq k, k+1 \\
\tilde{b}_{i k}=-b_{i, k+1}-\epsilon_{k} b_{i k} \phi\left(a_{k, k+1}\right)-a_{i k}^{\prime} b_{k, k+1} & i \neq k, k+1 \\
\tilde{b}_{k+1, i}=b_{k i} & i \neq k, k+1 \\
\tilde{b}_{i, k+1}=b_{i k} & \\
\tilde{b}_{k, k+1}=\mu b_{k+1, k} & \\
\tilde{b}_{k+1, k}=\mu^{-1} b_{k, k+1} & \\
\tilde{b}_{i j}=b_{i j} & i, j \neq k, k+1 \\
\tilde{C}=\Phi_{\sigma_{k}}^{L}\left(\epsilon_{k}^{-1} \phi_{B}(A)\right) \cdot C \cdot \Phi_{\sigma_{k}}^{R}(A)+\Theta_{k}^{L} \cdot A \cdot \Phi_{\sigma_{k}}^{R}(A) ; \\
\tilde{D}=\Phi_{\sigma_{k}}^{L}(A) \cdot D \cdot \Phi_{\sigma_{k}}^{R}\left(\epsilon_{k} \phi_{B}(A)\right)+\mu^{-1} \Phi_{\sigma_{k}}^{L}(A) \cdot A \cdot \Theta_{k}^{R} ; \\
\tilde{E}=\Phi_{\sigma_{k}}^{L}\left(\epsilon_{k}^{-1} \phi_{B}(A)\right) \cdot E \cdot \Phi_{\sigma_{k}}^{R}\left(\epsilon_{k} \phi_{B}(A)\right)-\Theta_{k}^{L} \cdot D \cdot \Phi_{\sigma_{k}}^{R}\left(\phi_{B}(A)\right)+\Theta_{k}^{L} \cdot \Theta_{k}^{R} ; \\
\tilde{F}=\Phi_{\sigma_{k}}^{L}\left(\epsilon_{k}^{-1} \phi_{B}(A)\right) \cdot F \cdot \Phi_{\sigma_{k}}^{R}\left(\epsilon_{k} \phi_{B}(A)\right)+\mu^{-1} \Phi_{\sigma_{k}}^{L}\left(\epsilon_{k}^{-1} \phi_{B}(A)\right) \cdot C \cdot \Theta_{k}^{R}+\Theta_{k}^{L} \cdot\left(1+\mu^{-1} A\right) \cdot \Theta_{k}^{R} .
\end{array}
$$

Here $\Phi_{\sigma_{k}}^{L}\left(\epsilon_{k}^{-1} \phi_{B}(A)\right)$ and $\Phi_{\sigma_{k}}^{R}\left(\epsilon_{k} \phi_{B}(A)\right)$ are the matrices which coincide with the $n \times n$ identity matrix except in the intersection of rows $k, k+1$ and columns $k, k+1$, where they are $\left(\begin{array}{cc}-\epsilon_{k}^{-1} \phi_{B}\left(a_{k+1, k}\right) & -1 \\ 1 & 0\end{array}\right)$ and $\left(\begin{array}{cc}-\epsilon_{k} \phi_{B}\left(a_{k, k+1}\right) & 1 \\ -1 & 0\end{array}\right)$, respectively, and $\Theta_{k}^{L}$ and $\Theta_{k}^{R}$ are the $n \times n$ matrices which are identically zero except in the $k k$ entry, where they are $-b_{k+1, k}$ and $-b_{k, k+1}$, respectively.

This identification of $\mathcal{A}$ and $\tilde{\mathcal{A}}$ gives the desired tame isomorphism. For anyone wishing to verify that $\partial$ and $\tilde{\partial}$ are intertwined by this identification, it may be helpful to note the following identities: $\Phi_{\sigma_{k}}^{L}\left(\epsilon_{k}^{-1} \phi_{B}(A)\right) \Lambda=$ $\tilde{\Lambda} \Phi_{\sigma_{k}}^{L}\left(\phi_{B}(A)\right), \Phi_{\sigma_{k}}^{R}\left(\epsilon_{k} \phi_{B}(A)\right) \tilde{\Lambda}=\Lambda \Phi_{\sigma_{k}}^{R}\left(\phi_{B}(A)\right)$, and

$$
\begin{aligned}
\tilde{B}= & \Phi_{\sigma_{k}}^{L}\left(\epsilon_{k}^{-1} \phi_{B}(A)\right) \cdot B \cdot \Phi_{\sigma_{k}}^{R}\left(\epsilon_{k} \phi_{B}(A)\right)+\mu^{-1} \Phi_{\sigma_{k}}^{L}(A) \cdot A \cdot \Theta_{k}^{R}(A) \\
& +\Theta_{k}^{L}(A) \cdot A \cdot \Phi_{\sigma_{k}}^{R}\left(\epsilon_{k} \phi_{B}(A)\right)+\partial\left(\Theta_{k}^{L}(A) \cdot \Theta_{k}^{R}(A)\right) .
\end{aligned}
$$

Positive stabilization. Here $\tilde{B}$ is obtained by adding a strand labeled 0 to $B$ and setting $\tilde{B}=B \sigma_{0}$. We will denote the framed knot DGA of $B$ by $(\mathcal{A}, \partial)$ as usual. Write the generators of the framed knot DGA of $\tilde{B}$ as $\tilde{a}_{i j}, \tilde{b}_{i j}, \tilde{c}_{i j}, \tilde{d}_{i j}, \tilde{e}_{i}$, where now $0 \leq i, j \leq n$; in the definition of the differential, the matrix $\Lambda$ now has $\lambda$ as its $(0,0)$ entry. Let $(\tilde{\mathcal{A}}, \tilde{\partial})$ denote the result of replacing $\lambda$ by $\lambda / \mu$ in the framed knot DGA of $\tilde{B}$.

We want an identification between $\mathcal{A}$, suitably stabilized as in the proof of Ng2, Thm. 2.10], and $\tilde{\mathcal{A}}$, which yields a stable tame isomorphism between $(\mathcal{A}, \partial)$ and $(\tilde{\mathcal{A}}, \tilde{\partial})$. It is given as follows: $a_{i_{1} i_{2}}=\tilde{a}_{i_{1} i_{2}}$ for $1 \leq i, j \leq n$;

$$
\begin{aligned}
b_{1 i} & =-\mu \tilde{b}_{0 i}+\tilde{b}_{1 i}-\mu \tilde{b}_{00} a_{0 i} & c_{i 1} & =-\mu^{-1} \tilde{c}_{i 0}+\tilde{c}_{i 1}-\mu^{-1} a_{i 0} \tilde{c}_{00} \\
b_{j i} & =\tilde{b}_{j i} & c_{i j} & =\tilde{c}_{i j}
\end{aligned}
$$


for $i \geq 1$ and $j \geq 2$;

$$
\begin{aligned}
d_{11} & =\tilde{d}_{00}-\mu \tilde{d}_{01}+\tilde{d}_{11}-\mu^{-1} \tilde{d}_{10}+\tilde{b}_{00} \tilde{c}_{01}+\mu^{-1} \tilde{b}_{10} \tilde{c}_{00} \\
d_{1 j} & =-\mu \tilde{d}_{0 j}+\tilde{d}_{1 j}+\tilde{b}_{00} \tilde{c}_{0 j} \\
d_{j 1} & =-\mu^{-1} \tilde{d}_{j 0}+\tilde{d}_{j 1}+\mu^{-1} \tilde{b}_{j 0} \tilde{c}_{00} \\
d_{j_{1} j_{2}} & =\tilde{d}_{j_{1} j_{2}} \\
e_{1} & =\tilde{e}_{0}+\tilde{e}_{1}-\mu \tilde{d}_{01}+\tilde{b}_{00} \tilde{c}_{01} \\
e_{j} & =\tilde{e}_{j} \\
d_{00} & =\mu^{-1} \tilde{d}_{10}-\tilde{d}_{11}+\mu \tilde{d}_{01}-\mu^{-1} \tilde{b}_{10} \tilde{c}_{00}-\tilde{b}_{00} \tilde{c}_{01} \\
e_{0} & =\mu \tilde{d}_{01}-\tilde{e}_{1}-\tilde{b}_{00} \tilde{c}_{01} \\
d_{10} & =\tilde{d}_{11}-\tilde{e}_{1} \quad d_{01}=\tilde{e}_{1} \quad d_{0 j}=\tilde{d}_{1 j} \quad d_{j 0}=\tilde{d}_{j 1}
\end{aligned}
$$

for $j, j_{1}, j_{2} \geq 2$; and

$$
\begin{aligned}
& b_{10}=\tilde{b}_{10}+\tilde{c}_{11} \quad \text { and } \quad c_{01}=\tilde{c}_{01}+\tilde{b}_{11} ; \\
& b_{00}=\tilde{b}_{00}-b_{11}-\lambda \Phi_{1 \ell}^{L} \tilde{c}_{\ell 1} \quad \text { and } \quad c_{00}=\tilde{c}_{00}-c_{11}-\lambda^{-1} \tilde{b}_{1 \ell} \Phi_{\ell 1}^{R} \text {; } \\
& b_{j 0}=\tilde{b}_{j 0}+\tilde{c}_{j 1}-b_{j 1}-\Phi_{j \ell}^{L} \tilde{c}_{\ell 1} \quad \text { and } \quad c_{0 j}=\tilde{c}_{0 j}+\tilde{b}_{1 j}-c_{1 j}-\tilde{b}_{1 \ell} \Phi_{\ell j}^{R} \text {; } \\
& b_{0 i}=\tilde{b}_{1 i} \quad \text { and } \quad c_{i 0}=\tilde{c}_{i 1}
\end{aligned}
$$

for $i \geq 1$ and $j \geq 2$. If we set $\partial b_{0 i}=-\mu a_{0 i}+a_{1 i}^{\prime}, \partial c_{i 0}=-a_{i 0}+a_{i 1}^{\prime}$, $\partial e_{0}=b_{00}, \partial d_{00}=b_{00}-c_{00}, \partial d_{i 0}=-b_{i 0}, \partial d_{0 i}=c_{0 i}$ for $i \geq 1$, then the above identification produces $\partial=\tilde{\partial}$.

Negative stabilization. Here $\tilde{B}=B \sigma_{0}^{-1}$; let $(\mathcal{A}, \partial)$ be the framed knot DGA of $B$, and let $(\tilde{\mathcal{A}}, \tilde{\partial})$ be the framed knot DGA of $\tilde{B}$ with $\lambda$ replaced by $\lambda \mu$, with notation as in the previous case.

In this case, the identification between $\mathcal{A}$ and $\tilde{\mathcal{A}}$ is as follows: $a_{i_{1} i_{2}}=\tilde{a}_{i_{1} i_{2}}$ for $1 \leq i, j \leq n$;

$$
\begin{aligned}
& b_{1 i}=\tilde{b}_{1 i}-\tilde{b}_{0 i}+\lambda \tilde{c}_{00} \Phi_{1 \ell}^{L} a_{\ell i} \quad c_{i 1}=\tilde{c}_{i 1}-\tilde{c}_{i 0}+\frac{1}{\lambda \mu} a_{i \ell} \Phi_{\ell 1}^{R} \tilde{b}_{00} \\
& b_{j i}=\tilde{b}_{j i} \quad c_{i j}=\tilde{c}_{i j} \text {; } \\
& d_{11}=-\tilde{d}_{10}+\tilde{d}_{11}-\tilde{d}_{01}+\tilde{d}_{00}-\frac{1}{\lambda \mu} b_{1 \ell} \Phi_{\ell 1}^{R} \tilde{b}_{00}-\lambda \tilde{c}_{00} \Phi_{1 \ell}^{L} c_{\ell 1}-\frac{\mu+1}{\mu} \tilde{c}_{00} \tilde{b}_{00} \\
& d_{1 j}=\tilde{d}_{1 j}-\tilde{d}_{0 j}-\lambda \tilde{c}_{00} \Phi_{1 \ell}^{L} c_{\ell j} \\
& d_{j 1}=\tilde{d}_{j 1}-\tilde{d}_{j 0}-\frac{1}{\lambda \mu} b_{j \ell} \Phi_{\ell 1}^{R} \tilde{b}_{00} \\
& d_{j_{1} j_{2}}=\tilde{d}_{j_{1} j_{2}} \\
& e_{1}=-\tilde{d}_{01}+\tilde{e}_{0}+\tilde{e}_{1}-\lambda \tilde{c}_{00} \Phi_{1 \ell}^{L} c_{\ell 1}-\tilde{c}_{00} \tilde{b}_{00}+\lambda \mu\left(\tilde{e}_{0}-\tilde{d}_{00}\right) \phi_{B}\left(a_{10}\right)+\frac{1}{\lambda \mu} \phi_{B}\left(a_{01}\right) \tilde{e}_{0} \\
& e_{j}=\tilde{e}_{j} \\
& d_{00}=\tilde{d}_{00} \quad e_{0}=\tilde{e}_{0} \quad d_{10}=\tilde{d}_{11}-\tilde{e}_{1} \quad d_{01}=\tilde{e}_{1} \quad d_{j 0}=\tilde{d}_{j 0} \quad d_{0 j}=\tilde{d}_{0 j} ;
\end{aligned}
$$




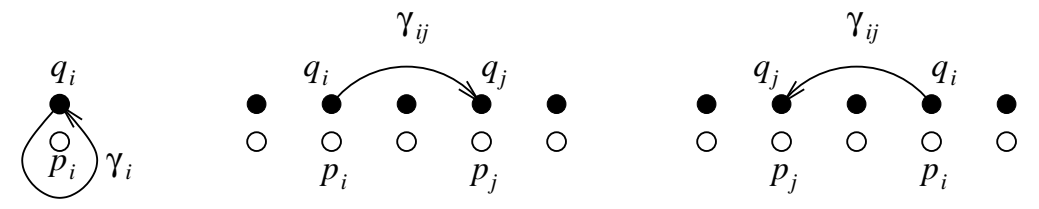

Figure 3 . The framed $\operatorname{arcs} \gamma_{i}$ (left) and $\gamma_{i j}$ for $i<j$ (middle) and $i>j$ (right).

$$
\begin{array}{rlrl}
b_{10}= & -\tilde{b}_{10}+c_{11}+\tilde{c}_{10}-\frac{1}{\lambda \mu} a_{1 \ell}^{\prime} \Phi_{\ell 1}^{R} \tilde{b}_{00} & & c_{01}=-\tilde{c}_{01}+b_{11}+\tilde{b}_{01}-\lambda \tilde{c}_{00} \Phi_{1 \ell}^{L} a_{\ell 1}^{\prime} \\
& -\left\{b_{1 \ell} \Phi_{\ell 1}^{R}+\tilde{b}_{0 \ell} \Phi_{\ell 1}^{R}\right. & \text { and } & -\phi_{B}\left(a_{01}\right)\left\{\Phi_{1 \ell}^{L} c_{\ell 1}\right. \\
& \left.+\lambda(1+\mu) \tilde{c}_{00}\right\} \phi_{B}\left(a_{10}\right) & & \\
& & & \left.+\Phi_{1 \ell}^{L} \tilde{c}_{\ell 0}+\frac{1+\mu}{\lambda \mu} \tilde{b}_{00}\right\} ; \\
b_{00}= & \tilde{b}_{00}+\lambda \mu \Phi_{1 \ell}^{L} \tilde{c}_{\ell 0} & \text { and } & c_{00}=\tilde{c}_{00}+\frac{1}{\lambda \mu} \tilde{b}_{0 \ell} \Phi_{\ell 1}^{R} ; \\
b_{j 0}=-\tilde{b}_{j 0}+\tilde{c}_{j 0}+\frac{1}{\lambda \mu} b_{j \ell} \Phi_{\ell 1}^{R}-\Phi_{j \ell}^{L} \tilde{c}_{\ell 0} & \text { and } & c_{0 j}=-\tilde{c}_{0 j}+\tilde{b}_{0 j}+\lambda \mu \Phi_{1 \ell}^{L} c_{\ell j}-\tilde{b}_{0 \ell} \Phi_{\ell j}^{R} ; \\
b_{0 i}=\tilde{b}_{0 i} & \text { and } \quad c_{i 0}=\tilde{c}_{i 0} .
\end{array}
$$

Then $\partial=\tilde{\partial}$ as before, once we set $\partial b_{0 i}=\mu a_{0 i}-\lambda \mu \Phi_{1 \ell}^{L} a_{\ell i}, \partial c_{i 0}=a_{i 0}-$ $\frac{1}{\lambda \mu} a_{i \ell} \Phi_{\ell 1}^{R}, \partial d_{00}=b_{00}-c_{00}, \partial e_{0}=b_{00}, \partial d_{i 0}=-b_{i 0}, \partial d_{0 i}=c_{0 i}$.

3.2. Proof of Theorem 2.10. This proof is essentially the proof of Theorem 1.3 from $\mathrm{Ng} 3$, with only slight modifications. Rather than giving the proof in full, we will outline the steps here and refer the reader to $[\mathrm{Ng} 3$ for details.

Let $D$ be the unit disk in $\mathbb{C}$, and choose points $p_{1}, \ldots, p_{n}, q_{1}, \ldots, q_{n}$ in $D$ such that $p_{1}, \ldots, p_{n}$ lie on the real line with $p_{1}<\cdots<p_{n}$ and $q_{j}=p_{j}+\epsilon i$ for each $j$ and some small $\epsilon>0$. We will depict the points $p_{1}, \ldots, p_{n}$ by an open circle, and $q_{1}, \ldots, q_{n}$ by closed circles; see Figure 3

The braid group $B_{n}$ acts by homeomorphisms in the usual way on the punctured disk $D \backslash P$, where $P$ denotes $\left\{p_{1}, \ldots, p_{n}\right\}$. That is, $\sigma_{k}$ rotates $p_{k}, p_{k+1}$ in a counterclockwise fashion around each other until they change places. Furthermore, we can stipulate that the line segments $p_{k} q_{k}$ and $p_{k+1} q_{k+1}$ remain vertical and of fixed length throughout the isotopy, so that $\sigma_{k}$ also interchanges $q_{k}, q_{k+1}$, and hence every element of $B_{n}$ fixes the set $Q=\left\{q_{1}, \ldots, q_{n}\right\}$. Then $B_{n}$ acts on the set of framed cords, as defined below.

Definition 3.3 (cf. Ng3, Def. 3.1]). A framed cord of $(D, P, Q)$ is a continuous map $\gamma:[0,1] \rightarrow D \backslash P$ such that $\gamma(0), \gamma(1) \in Q$. The set of framed arcs modulo homotopy is denoted $\mathcal{P}_{n}$. Define $\delta_{i}, 1 \leq i \leq n$, and $\gamma_{i j}$, $1 \leq i \neq j \leq n$, to be the framed cords depicted in Figure 3

Proposition 3.4 (cf. Ng3, Props. 2.2, 3.2, 3.3]). With $\mathcal{A}_{n}$ as in Section 2.3. there is an unique map $\psi: \mathcal{P}_{n} \rightarrow \mathcal{A}_{n}$ satisfying:

(1) Equivariance: $\psi(B \cdot \gamma)=\phi_{B}(\psi(\gamma))$ for any $B \in B_{n}$ and $\gamma \in \mathcal{P}_{n}$, where $B \cdot \gamma$ denotes the action of $B$ on $\gamma$; 

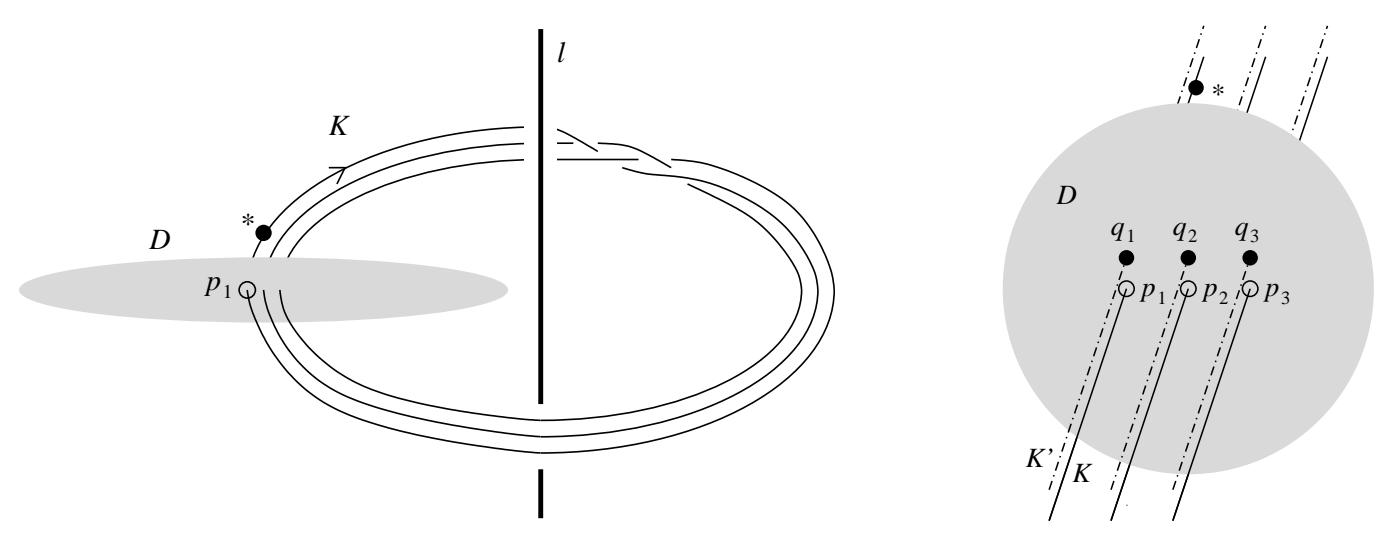

FiguRE 4 . Wrapping a braid around the line $\ell$ to create the knot $K$, and a close-up of the area around the disk $D$.

(2) Framing: if $\gamma$ is a framed arc with $\gamma(0)=p_{j}$, then $\psi\left(\gamma_{j} \gamma\right)=\mu \psi(\gamma)$, where $\gamma_{j} \gamma$ denotes the framed arc obtained by concatenating $\gamma_{j}$ and $\gamma m$ and similarly if $\gamma(1)=p_{l}$, then $\psi\left(\gamma \gamma_{l}\right)=\mu \psi(\gamma)$;

(3) Normalization: $\psi\left(\gamma_{i j}\right)=-a_{i j}$ if $i<j, \psi\left(\gamma_{i j}\right)=-\mu a_{i j}$ if $i>j$, and $\psi\left(\gamma_{i}^{0}\right)=1+\mu$ where $\gamma_{i}^{0}$ is the trivial cord which is constant at $q_{i}$.

Furthermore, we have the following skein relations for framed cords:

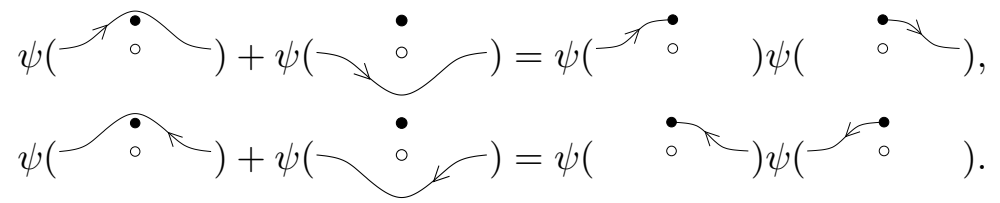

Finally, the above skein relations, along with (2) and (3), suffice to define $\psi$.

Note that the normalization of (3) has been chosen for compatibility with (11) and (2): $\sigma_{k}$ sends $\gamma_{k, k+1}$ to $\underset{0 p_{k}}{q_{k}} \overbrace{q_{k+1}}$, which is mapped under $\psi$ to $\mu^{-1} \psi\left(\gamma_{k+1, k}\right)$. The main difference between Proposition 3.4 and the corresponding results from $\mathrm{Ng} 3$ is the framing axiom, which incorporates the variable $\mu$ into the map $\psi$.

Proof of Theorem [2.10 (cf. $[\mathrm{Ng} 3, \S 3.2]$ ). Suppose that the knot $K$ is the closure of a braid $B \in B_{n}$, with framing induced from the braid. If $\ell$ is a line in $\mathbb{R}^{3}$, then $\mathbb{R}^{3} \backslash \ell$ is topologically a solid torus $S^{1} \times D^{2}$; let $D$ be a $D^{2}$ fiber of this solid torus, viewed as sitting in $\mathbb{R}^{3}$. The complement of $D$ in $S^{1} \times D^{2}$ is a solid cylinder in which we can embed $B$, and the closure of this embedding in $\mathbb{R}^{3}$ is precisely $K$.

Now $K$ intersects $D$ in $n$ points $p_{1}, \ldots, p_{n}$, and the framing of $K$ allows us to push $K$ off of itself to obtain another knot $K^{\prime}$, which then intersects $D$ in points $q_{1}, \ldots, q_{n}$. Choose the base point $*$ on $K$, which we need to define the cord algebra, to be just beyond $p_{1}$ in the direction of the orientation of 
$K$. See Figure 4 For $1 \leq i \leq n$, let $\eta_{i}$ denote the path along $K^{\prime}$ running from $q_{i}$ to $q_{1}$ in the direction of the orientation of $K$ (so that it does not pass $*)$.

Any cord $\gamma$ of $K$, in the sense of Definition 2.3, can be homotoped to lie entirely in $D$; a slight perturbation of the ends yields a framed cord $\tilde{\gamma}$ of $(D, P, Q)$, in the sense of Definition 3.3 say beginning at $q_{i}$ and ending at $q_{j}$. Now the perturbation yielding $\tilde{\gamma}$ is not uniquely defined up to homotopy, and so $\psi(\tilde{\gamma})$ is defined in $\mathcal{A}_{n}$ only up to multiplication by powers of $\mu$. We can however compensate for this by considering the linking number of $K$ and the closed oriented curve $\tilde{\gamma} \cup \eta_{j} \cup\left(-\eta_{i}\right)$. More precisely, it is easy to check that $\mu^{\operatorname{lk}\left(K, \tilde{\gamma} \cup \eta_{j} \cup\left(-\eta_{i}\right)\right)} \psi(\tilde{\gamma})$ is well-defined. Given a cord of $K$ lying in $D$, we hence get an element of $\mathcal{A}_{n}$ which we denote by $\tilde{\psi}(\gamma)$. Moreover, the skein relation defining the cord algebra maps to the skein relation in Proposition 3.4

We conclude that the cord algebra of $K$ is a quotient of $\mathcal{A}_{n}$; this quotient arises from the non-uniqueness of the homotopy from an arbitrary cord of $K$ to a cord of $K$ lying in $D$. Now the argument of the proof of $\mathrm{Ng3}$, Thm. 1.3] shows that the cord algebra of $K$ is $\mathcal{A}_{n} / \mathcal{I}$, where $\mathcal{I}$ is the subalgebra of $\mathcal{A}_{n}$ generated by the entries of the matrices $\left(1-\Lambda \cdot \Phi_{B}^{L}\right) \cdot A$ and $A \cdot\left(1-\Phi_{B}^{R} \cdot \Lambda^{-1}\right)$. (The reason that we need the entries of $A$ above the diagonal to be of the form $\mu a_{i j}$, rather than $a_{i j}$ as in Ng3, is because in this case the map $\psi$ sends $\gamma_{i j}$ to $-\mu a_{i j}$ rather than $-a_{i j}$.) But $\mathcal{A}_{n} / \mathcal{I}$ is precisely the degree 0 homology of the framed knot DGA of $K$.

\section{Properties of the InVARiants}

4.1. Symmetries. The cord algebra and framed knot DGA invariants change in predictable manners when we change the framed knot by various elementary operations: framing change, mirror, and inversion. Here, as usual, "inversion" refers to reversing the orientation of the knot, and the framing under mirror and inversion is the induced framing from the original knot.

The effects of these operations are easiest to see in the homotopy interpretation of the framed cord algebra (Definition 2.2). As mentioned previously, changing the framing of a knot by $f$ keeps the meridian fixed and changes the longitude by the corresponding power of the meridian; hence the framed cord algebra changes by replacing $\lambda$ by $\lambda \mu^{f}$. A reflection of three-space about a plane changes a framed knot into its mirror, maps the knot's longitude to its mirror's longitude, and maps the meridian to the mirror's meridian with the opposite orientation; hence mirroring changes the framed cord algebra by replacing $\mu$ by $\mu^{-1}$. Changing the orientation of a framed knot changes the orientation of both its longitude and meridian, and so inversion replaces $\lambda$ by $\lambda^{-1}$ and $\mu$ by $\mu^{-1}$. 
Proposition 4.1. The framed cord algebra of a framed knot changes under the following operations as shown: framing change by $f, \lambda \rightarrow \lambda \mu^{f}$; mirror, $\mu \rightarrow \mu^{-1}$; inversion, $\lambda \rightarrow \lambda^{-1}$ and $\mu \rightarrow \mu^{-1}$. In addition, the commutative framed cord algebras (in which multiplication is commutative) of a knot and its inverse are isomorphic.

Proof. The only statement left to prove is the invariance of the commutative framed cord algebra under inversion. Let $l, m$ be the longitude and meridian of a framed knot $K$; then $l^{-1}, m^{-1}$ are the longitude and meridian of its inverse. The map sending $\gamma \in \pi_{1}\left(S^{3} \backslash K\right)$ to $\gamma^{-1}$ extends in a natural way to a map on the tensor algebra generated by $\pi_{1}\left(S^{3} \backslash K\right)$. This map descends to an isomorphism on the commutative framed cord algebras; the key point is that the relation $\left[\gamma_{1} \gamma_{2}\right]+\left[\gamma_{1} m \gamma_{2}\right]=\left[\gamma_{1} \gamma_{2}\right]$ in the cord algebra of $K$ is mapped to

$$
\left[\gamma_{2}^{-1} \gamma_{1}^{-1}\right]+\left[\gamma_{2}^{-1} m^{-1} \gamma_{1}^{-1}\right]=\left[\gamma_{1}\right]^{-1}\left[\gamma_{2}\right]^{-1}=\left[\gamma_{2}\right]^{-1}\left[\gamma_{1}\right]^{-1}
$$

which is the corresponding relation for the cord algebra of the inverse of $K$.

Not surprisingly, the same operations lead to the same transformations in $\lambda, \mu$ in the framed knot DGA, although the proof is not particularly enlightening. Define the commutative framed knot DGA of a knot to be the quotient of the framed knot DGA obtained by imposing sign-commutativity, i.e., $v w=(-1)^{|v||w|} w v$. (Note that this does not specialize to the "abelian knot DGA" from $\mathbb{N g 2}$ when we set $\lambda=\mu=1$; there does not seem to be a reasonable lift of the abelian knot DGA to group ring coefficients.)

Proposition 4.2. The framed knot DGA of a framed knot changes under framing change, mirroring, and inversion as in Proposition 4.1. and the commutative framed knot DGAs of a knot and its inverse are equivalent.

Proof. Framing change has already been covered by Theorem 2.7

Mirror: We follow the proof of Proposition 6.9 from $\mathrm{Ng} 2$, which is the analogous result for the DGA over $\mathbb{Z}$; see that proof for notation. If a framed knot $K$ is given as the closure of a braid $B \in B_{n}$, then the mirror of $K$ (with the corresponding framing) is the closure of $B^{*}$. Let $(\mathcal{A}, \partial)$ be the framed knot DGA for $B$, and let $(\tilde{\mathcal{A}}, \tilde{\partial})$ be the framed knot DGA for $B^{*}$, but with $\mu$ replaced by $\mu^{-1}$ and $\Lambda$ replaced by $\tilde{\Lambda}=\Lambda_{n}$, with notation as in Section 3.1 this is equivalent to the usual framed knot DGA for $B^{*}$ (with $\mu \rightarrow \mu^{-1}$ ) by Proposition 3.1 It suffices to show that $(\mathcal{A}, \partial)$ and $(\tilde{\mathcal{A}}, \tilde{\partial})$ are tamely isomorphic.

Assemble the generators $a_{i j}, \tilde{a}_{i j}$ into matrices $A, \tilde{A}$ as usual, except that $\tilde{A}$ has $\mu$ replaced by $\mu^{-1}$. If we set $\tilde{a}_{n+1-i, n+1-j}=a_{i j}$ as in the proof of $\mathrm{Ng} 2$, Prop. 6.9], then we find that $\tilde{A}=\mu^{-1} \Xi(A)$, while $\Phi_{B^{*}}^{L}(\tilde{A})=\Xi\left(\Phi_{B}^{L}(A)\right)$ and $\Phi_{B^{*}}^{R}(\tilde{A})=\Xi\left(\Phi_{B}^{R}(A)\right)$. Now identify the other generators of $\mathcal{A}$ and $\tilde{\mathcal{A}}$ by setting $\tilde{B}=\mu^{-1} \Xi(B), \tilde{C}=\mu^{-1} \Xi(C), \tilde{D}=\mu^{-1} \Xi(D), \tilde{e}_{i}=\mu^{-1} e_{n+1-i}$. Then $\partial \tilde{B}=\mu^{-1} \partial \Xi B=\mu^{-1} \Xi\left(\left(1-\Lambda \cdot \Phi_{B}^{L}(A)\right) \cdot A\right)=\left(1-\tilde{\Lambda} \cdot \Phi_{B^{*}}^{L}(\tilde{A})\right) \cdot \tilde{A}=\tilde{\partial} \tilde{B}$ 
and similarly $\partial=\tilde{\partial}$ on the other generators of $\mathcal{A}$.

Inverse: Let $(\mathcal{A}, \partial)$ be the framed knot DGA for $B$, and let $(\tilde{\mathcal{A}}, \tilde{\partial})$ be the framed knot DGA for $B^{-1}$, but with $\lambda$ replaced by $\lambda^{-1}$. Since the closure of $\left(B^{-1}\right)^{*}$ is the inverse of the closure of $B$, and the framed knot DGA for $\left(B^{-1}\right)^{*}$ is the framed knot DGA for $B^{-1}$ with $\mu$ replaced by $\mu^{-1}$ by the above proof for mirrors, it suffices to prove that $(\mathcal{A}, \partial)$ and $(\tilde{\mathcal{A}}, \tilde{\partial})$ are equivalent to conclude that the framed knot DGAs of a knot is equivalent to the framed knot DGA of its inverse after setting $\lambda \rightarrow \lambda^{-1}$ and $\mu \rightarrow \mu^{-1}$.

Assemble the generators of $\tilde{\mathcal{A}}$ into matrices $\tilde{A}$, etc., and let $\tilde{\Lambda}=\Lambda^{-1}$, so that $\tilde{\partial} \tilde{B}=\left(1-\tilde{\Lambda} \cdot \Phi_{B^{-1}}^{L}(\tilde{A})\right) \cdot \tilde{A}$ and so forth. Now set $\tilde{a}_{i j}=\phi_{B}\left(a_{i j}\right)$, which implies that $\tilde{A}=\Phi_{B}^{L}(A) \cdot A \cdot \Phi_{B}^{R}(A)$, and $\tilde{B}=-\Lambda^{-1} \cdot B \cdot \Phi_{B}^{R}(A)$, $\tilde{C}=-\Phi_{B}^{L}(A) \cdot C \cdot \Lambda, \tilde{D}=\Lambda^{-1} \cdot D \cdot \Lambda, \tilde{e}_{i}=d_{i i}-e_{i}$. Since $\Phi_{B^{-1}}^{L}(\tilde{A})=\left(\Phi_{B}^{L}(A)\right)^{-1}$ and $\Phi_{B^{-1}}^{R}(\tilde{A})=\left(\Phi_{B}^{R}(A)\right)^{-1}$, it is easy to check that this identification gives $\partial=\tilde{\partial}$; for instance,

$\tilde{\partial} \tilde{B}=\left(1-\Lambda^{-1} \cdot\left(\Phi_{B}^{L}(A)\right)^{-1}\right) \cdot \Phi_{B}^{L}(A) \cdot A \cdot \Phi_{B}^{R}(A)=-\Lambda^{-1} \cdot\left(1-\Lambda \cdot \Phi_{B}^{L}\right) \cdot A \cdot \Phi_{B}^{R}(A)=\partial \tilde{B}$.

It remains to show that the commutative framed knot DGAs of a knot and its inverse are equivalent. Because of the previous argument, it suffices to prove that the commutative framed knot DGA $(\mathcal{A}, \partial)$ of a braid $B$ is equivalent to the same commutative framed $\operatorname{knot} \operatorname{DGA}(\tilde{\mathcal{A}}, \tilde{\partial})$ with $\lambda, \mu$ replaced by $\lambda^{-1}, \mu^{-1}$. As usual, assemble the generators of $\tilde{\mathcal{A}}$ into matrices $\tilde{A}$, etc., except that $\tilde{A}$ has $\mu$ replaced by $\mu^{-1}$. If we set $\tilde{a}_{i j}=a_{j i}$ for all $i, j$, then $\tilde{A}=\mu^{-1} A^{t}$. Further set $\tilde{B}=\mu^{-1} C^{t}, \tilde{C}=\mu^{-1} B^{t}, \tilde{D}=-\mu^{-1} D^{t}$, $e_{i}=\mu^{-1}\left(e_{i}-d_{i i}\right)$. Now since the $a_{i j}$ commute with each other, Proposition 6.1 from Ng2 implies that $\Phi_{B}^{L}(\tilde{A})=\left(\Phi_{B}^{R}(A)\right)^{t}$ and $\Phi_{B}^{R}(\tilde{A})=\left(\Phi_{B}^{L}(A)\right)^{t}$. It follows easily that $\partial=\tilde{\partial}$; for instance,

$\tilde{\partial} \tilde{B}=\left(1-\Lambda^{-1} \cdot \Phi_{B}^{L}(\tilde{A})\right) \cdot \tilde{A}=\mu^{-1}\left(1-\Lambda^{-1} \cdot\left(\Phi_{B}^{R}(A)\right)^{t}\right) \cdot A^{t}=\mu^{-1}\left(A \cdot\left(1-\Phi_{B}^{R}(A)\right)\right)^{t}=\partial \tilde{B}$,

as claimed.

Although the commutative framed knot DGA does not distinguish between knots and their inverses, it is conceivable that the usual noncommutative framed knot DGA, or the cord algebra, might. Presumably an application to distinguish inverses would require some sort of involved calculation using noncommutative Gröbner bases.

4.2. Relation to the alternate knot DGA. It is immediate from the definition of the framed knot DGA that one can recover the knot DGA over $\mathbb{Z}$ from $\overline{N g 2}$ by setting $\lambda=\mu=1$. What is less obvious is that the framed knot DGA also specializes to the "alternate knot DGA" from [Ng2, §9].

Proposition 4.3. The knot DGA is obtained from the framed knot DGA by setting $\lambda=\mu=1$; the alternate knot DGA is obtained from the framed knot DGA by setting $\lambda=\mu=-1$. 
Proof. Let $B \in B_{n}$ be a braid whose closure gives the desired framed knot, and note that, since $B$ closes to a knot, $w(B) \equiv n+1(\bmod 2)$. Setting $\lambda=\mu=-1$ in the framed knot DGA for $K$ is the same as setting $\mu=-1$, $\lambda=(-1)^{w(B)+1}=(-1)^{n}$ in the framed knot DGA corresponding to $B$. The argument of the proof of Proposition 3.1 shows that $\Lambda$ can be replaced in the definition of the framed knot DGA by any diagonal matrix, the product of whose diagonal entries is $\lambda$. Since $\lambda=(-1)^{n}$, we can use the matrix -1 instead of $\Lambda$ in defining the framed knot DGA. Let $(\mathcal{A}, \partial)$ denote the resulting DGA, let $(\tilde{\mathcal{A}}, \tilde{\partial})$ denote the alternate knot DGA of $B$, and assemble the generators of $\mathcal{A}$ and $\tilde{\mathcal{A}}$ into matrices, as usual.

If we set $a_{i j}=\tilde{a}_{i j}$ if $i<j$ and $a_{i j}=-\tilde{a}_{i j}$ if $i>j$, then $A=-\tilde{A}$. In the notation of Section 9.1 of $[\mathrm{Ng} 2$, since $\phi, \tilde{\phi}$ are conjugate through the automorphism which negates $a_{i j}$ for $i>j$, we have $\tilde{\Phi}_{B}^{L}(\tilde{A})=\Phi_{B}^{L}(A)$ and $\tilde{\Phi}_{B}^{R}(\tilde{A})=\Phi_{B}^{R}(A)$. It follows readily from the definition of the alternate knot DGA that if we further set $\tilde{B}=-B, \tilde{C}=-C, \tilde{D}=-D, \tilde{e}_{i}=-e_{i}$, then $\partial=\tilde{\partial}$. The proposition follows.

Note that there are, in fact, four natural projections of the framed knot DGA to an invariant over $\mathbb{Z}$, corresponding to sending each of $\lambda, \mu$ to \pm 1 . We have seen that two of these projections yield the knot DGA and the alternate knot DGA from Ng2. The other two seem to behave slightly less nicely. For instance, for two-bridge knots, it can be shown that both the knot DGA and the alternate knot DGA have degree 0 homology which can be written as a quotient of $\mathbb{Z}[x]$ by a principal ideal. (For the knot DGA, this was shown in $\mathrm{Ng2}$, Thm. 7.1].) This is not the case for the other two projections.

As an example, consider the left-handed trefoil examined in Section 2.3 For $\lambda=\mu=1$, we obtain $H C_{0} \cong \mathbb{Z}[x] /\left(x^{2}+x-2\right)$, the knot DGA for the trefoil, and for $\lambda=\mu=-1, H C_{0} \cong \mathbb{Z}[x] /\left(x^{2}-x\right)$, the alternate knot DGA for the trefoil. By contrast, we have $H C_{0} \cong \mathbb{Z}[x] /\left(x^{2}-x, 2 x\right)$ when $\lambda=1$ and $\mu=-1$, and $H C_{0} \cong \mathbb{Z}[x] /\left(x^{2}+x-2,2 x\right)$ when $\lambda=-1$ and $\mu=1$.

It may be worth noting that, by Proposition 4.2. none of the projections to $\mathbb{Z}$ can detect mirrors or inverses, since $\lambda=\lambda^{-1}$ and $\mu=\mu^{-1}$ in all cases. We also remark that the "special" status of the cases $\lambda=\mu=1$ and $\lambda=\mu=-1$ is an artifact of a choice of a particular spin structure on $T^{2}$. More precisely, the signs in the definition of the framed knot DGA, which arise from a coherent choice of orientations of the relevant moduli spaces, depend on a choice of one of the (four) spin structures on the Legendrian 2-torus; changing the spin structure negates either or both of $\lambda$ and $\mu$.

\subsection{Framed knot contact homology and the Alexander polynomial.} It has been demonstrated in $[\mathrm{Ng} 3, \$ 7.2]$ that a linearized version of knot contact homology over $\mathbb{Z}$ encodes the determinant of the knot. Here we 
show that the framed knot DGA has a similar natural linearization, with respect to which we can deduce the Alexander polynomial of the knot.

Let $K$ be a knot, and let $(\mathcal{A}, \partial)$ denote the DGA over $\mathbb{Z}\left[\mu^{ \pm 1}\right]$ obtained by setting $\lambda=1$ in the framed knot DGA of $K$. To define a linearization of $(\mathcal{A}, \partial)$, we need an augmentation of $(\mathcal{A}, \partial)$ over $\mathbb{Z}\left[\mu^{ \pm 1}\right]$, that is, an algebra map $\epsilon: \mathcal{A} \rightarrow \mathbb{Z}\left[\mu^{ \pm 1}\right]$ which is 0 in nonzero degrees and satisfies $\epsilon \circ \partial=0$. In this case, an augmentation is just a ring homomorphism from the cord algebra of $K$ with $\lambda=1$ to $\mathbb{Z}\left[\mu^{ \pm 1}\right]$. There is a natural choice for such a homomorphism, namely the one which sends every cord to $1+\mu$; this clearly satisfies the relations for the cord algebra.

If $\epsilon$ denotes the corresponding augmentation, which we call the distinguished augmentation of the framed knot DGA, then we can construct a linearized version of $(\mathcal{A}, \partial)$ in the standard way. Let $\mathcal{M}$ be the subalgebra of $\mathcal{A}$ generated by all words (in $a_{i j}, b_{i j}, c_{i j}, d_{i j}, e_{i}$ ) of length at least 1 , and let $\varphi_{\epsilon}: \mathcal{A} \rightarrow \mathcal{A}$ be the algebra isomorphism sending each generator $g$ of $\mathcal{A}$ to $g+\epsilon(g)$. The differential $\varphi_{\epsilon} \circ \partial \circ \varphi_{\epsilon}^{-1}$ maps $\mathcal{M}$ to itself and hence descends to a differential $\partial_{\epsilon}^{\text {lin }}$ on the free $\mathbb{Z}\left[\mu^{ \pm 1}\right]$-module $\mathcal{M} / \mathcal{M}^{2}$. We define the linearized framed contact homology of $K$, written $H C_{*}^{\operatorname{lin}}(K)$, to be the graded homology of $\partial_{\epsilon}^{\text {lin }}$.

To relate linearized homology to the Alexander polynomial, we recall some elementary knot theory adapted from [Rol. Fix a knot $K$ in $S^{3}$, and let $\tilde{X}_{K}$ denote the infinite cyclic cover of the knot complement. Then $H_{1}\left(\tilde{X}_{K}\right)$ has a natural $\mathbb{Z}\left[t^{ \pm 1}\right]$ module structure, with respect to which it is called the Alexander invariant. The Alexander polynomial $\Delta_{K}(t)$ can be deduced from the Alexander invariant in the usual way; in particular, if the Alexander invariant is cyclic of the form $\mathbb{Z}\left[t^{ \pm 1}\right] /(p(t))$, then $p(t)$ is the Alexander polynomial up to units.

Proposition 4.4. If $K$ is a knot, then we have an isomorphism of $\mathbb{Q}\left[\mu^{ \pm 1}\right]$ modules

$$
H C_{1}^{\text {lin }}(K) \otimes \mathbb{Q} \cong\left(H_{1}\left(\tilde{X}_{K}\right) \oplus \mathbb{Q}\left[\mu^{ \pm 1}\right]\right) \otimes\left(H_{1}\left(\tilde{X}_{K}\right) \oplus \mathbb{Q}\left[\mu^{ \pm 1}\right]\right) \oplus\left(\mathbb{Q}\left[\mu^{ \pm 1}\right]\right)^{m}
$$

for some $m \geq 0$. Here $H_{1}\left(\tilde{X}_{K}\right)$ is understood to be a $\mathbb{Q}\left[\mu^{ \pm 1}\right]$-module by setting $t=-\mu$.

Before proving for Proposition 4.4 we note that the result, along with some simple linear algebra, implies that we can calculate the Alexander polynomial from $H C_{1}^{\text {lin }}$.

Corollary 4.5. The framed knot DGA with $\lambda=1$, along with the distinguished augmentation of the DGA, determines the Alexander polynomial.

Proof. Suppose that the invariant factors for the $\mathbb{Q}\left[\mu^{ \pm 1}\right]$-module $H_{1}\left(\tilde{X}_{K}\right)$ are $p_{1}, \ldots, p_{k} \in \mathbb{Q}\left[\mu^{ \pm 1}\right]$, with $p_{1}|\cdots| p_{k}$, so that $p_{1} \ldots p_{k}$ is some rational multiple of the Alexander polynomial. Then the invariant factors of $\left(H_{1}\left(\tilde{X}_{K}\right) \oplus \mathbb{Q}\left[\mu^{ \pm 1}\right]\right) \otimes\left(H_{1}\left(\tilde{X}_{K}\right) \oplus \mathbb{Q}\left[\mu^{ \pm 1}\right]\right) \oplus\left(\mathbb{Q}\left[\mu^{ \pm 1}\right]\right)^{m}$ are $\operatorname{gcd}\left(p_{i}, p_{j}\right)$ for $1 \leq i, j \leq k$, along with $2 k$ additional invariant factors which consist of 


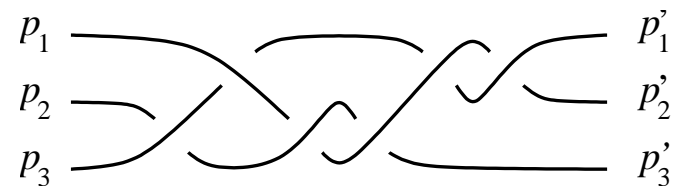

Figure 5. The braid $B=\sigma_{1}^{2} \sigma_{2}^{2} \sigma_{1}^{-1} \sigma_{2}$, with $v_{B}=\left(1, \mu^{2}, 1\right)$ and $u_{B}=\left(\mu^{2}, \mu^{2}, 1\right)$.

$p_{1}, \ldots, p_{k}$ with multiplicity 2 each. In other words, the invariant factors of $H C_{1}^{\operatorname{lin}}(K)$ are $p_{1}$ with multiplicity $2 k+1, p_{2}$ with multiplicity $2 k-1, \ldots, p_{k}$ with multiplicity 3 . From $H C_{1}^{\text {lin }}(K)$ we can hence deduce $p_{1}, \ldots, p_{k}$. This gives us the Alexander polynomial up to a constant factor, which can be eliminated via the normalization $\Delta_{K}(1)=1$.

The proof of Proposition 4.4 will occupy the remainder of this section. It is nearly identical to the proof of the analogous Proposition 7.8 from $\mathrm{Ng} 2$ and is rather unilluminating, and the reader may wish to skip it.

We first give an explicit description of the distinguished augmentation for a framed knot DGA, as derived by inspecting the translation between cords and framed knot contact homology from the proof of Theorem 2.10. Let $B \in B_{n}$ be a braid which closes to a knot. Draw $B$ from right to left with $\sigma_{k}$ giving a crossing where strand $k$ crosses over strand $k+1$, viewed from the right; see Figure 5. Write $p_{1}, \ldots, p_{n}$ for the leftmost endpoints of the braid, and $p_{1}^{\prime}, \ldots, p_{n}^{\prime}$ for the rightmost endpoints. For $1 \leq i \leq n$, traverse the braid from left to right, beginning at $p_{i}$ and looping around (that is, identify $p_{j}^{\prime}$ with $p_{j}$ ) until $p_{1}$ is reached; this traces out a path on the braid, which we define to be trivial when $i=1$. Now let $r_{i}$ be the signed number of undercrossings traversed by this path, where an undercrossing contributes 1 or -1 depending on whether it corresponds to a braid generator $\sigma_{k}$ or $\sigma_{k}^{-1}$. Finally, define $v_{B}$ to be the vector $\left(\mu^{r_{1}}, \ldots, \mu^{r_{n}}\right)$.

Recall from Section 3.1 that $\Delta(v)$ is a diagonal matrix whose diagonal entries are the entries of $v$, and let $\Theta$ denote the $n \times n$ matrix whose entries are all $-\mu-1$.

Definition 4.6. The distinguished augmentation of the framed knot DGA of a braid $B \in B_{n}$ is the map which sends

$$
A \mapsto \Delta\left(v_{B}\right) \cdot \Theta \cdot \Delta\left(v_{B}\right)^{-1}
$$

in other words, it sends $a_{i j}$ to $\mu^{r_{i}-r_{j}}(-\mu-1)$ if $i>j$ and $\mu^{r_{i}-r_{j}-1}(-\mu-1)$ if $i<j$.

We will see shortly that the distinguished augmentation is, in fact, an augmentation.

A bit more notation: let $\Lambda_{B}$ denote the matrix $\Lambda$ with $\lambda$ set to $\mu^{-w(B)}$ where $w(B)$ is the writhe of $B$; that is, $\Lambda_{B}=\Delta\left(\left(\mu^{-w(B)}, 1, \ldots, 1\right)\right)$. Define 
the vector $u_{B}$ to be $\left(\mu^{s_{1}}, \ldots, \mu^{s_{n}}\right)$, where $s_{i}$ is the signed number of undercrossings traversed if we begin at $p_{i}$, travel from left to right, and end when we reach the right hand end of the braid; see Figure 5.

Also, let $\hat{B}$ be the braid obtained by reversing the word which gives $B$. Finally, let Bur denote the Burau representation on $B_{n}$, defined over $\mathbb{Z}\left[t^{ \pm 1}\right]$, so that $\operatorname{Bur}_{\sigma_{k}}$ is the $n \times n$ matrix which is the identity except from the $2 \times 2$ submatrix formed by the $k, k+1$ rows and columns, which is $\left(\begin{array}{cc}1-t & t \\ 1 & 0\end{array}\right)$. We now give a series of lemmas, culminating in the proof of Proposition 4.4.

Lemma 4.7. Let $v_{k}$ denote the vector $(1, \ldots, 1, \mu, 1, \ldots, 1)$, with $\mu$ in the $k$-th position. Then

$$
\begin{aligned}
\phi_{\sigma_{k}}(\Theta) & =\Delta\left(v_{k}\right) \cdot \Theta_{k} \cdot \Delta\left(v_{k}\right)^{-1}, \\
\Phi_{\sigma_{k}}^{L}(\Theta) & =\Delta\left(v_{k}\right) \cdot\left(\left.\operatorname{Bur}_{\sigma_{k}}\right|_{t=-1 / \mu}\right), \\
\Phi_{\sigma_{k}}^{R}(\Theta) & =\left(\left.\operatorname{Bur}_{\sigma_{k}}\right|_{t=-\mu}\right)^{T} \cdot \Delta\left(v_{k}\right)^{-1},
\end{aligned}
$$

where $^{T}$ denotes transpose.

Proof. Easy calculation using the definitions, along with the expressions for $\Phi_{\sigma_{k}}^{L}$ and $\Phi_{\sigma_{k}}^{R}$ from Ng2, Lemma 4.6].

Lemma 4.8. We have

$\Phi_{B}^{L}(\Theta)=\Delta\left(u_{B}\right) \cdot\left(\left.\operatorname{Bur}_{\hat{B}}\right|_{t=-1 / \mu}\right)=\Lambda_{B}^{-1} \cdot \Delta\left(s(B) v_{B}\right)^{-1} \cdot \Delta\left(v_{B}\right) \cdot\left(\left.\operatorname{Bur}_{\hat{B}}\right|_{t=-1 / \mu}\right)$

and

$\Phi_{B}^{R}(\Theta)=\left(\left.\operatorname{Bur}_{\hat{B}}\right|_{t=-\mu}\right) \cdot \Delta\left(u_{B}\right)^{-1}=\left(\left.\operatorname{Bur}_{\hat{B}}\right|_{t=-\mu}\right) \cdot \Delta\left(v_{B}\right)^{-1} \cdot \Delta\left(s(B) v_{B}\right) \cdot \Lambda_{B}$,

with $s(B)$ defined as in Section 3.1.

Proof. We will establish the formula for $\Phi_{B}^{L}(\Theta)$, with $\Phi_{B}^{R}(\Theta)$ established similarly. The second equality follows from the fact that $s_{i}=r_{i}-r_{s(B)(i)}$ for $i \geq 2$ and $s_{1}=r_{1}-r_{s(B)(1)}+w(B)$. To prove the first equality, we use induction, noting that $u_{B}$ can be defined even when $B$ closes to a multicomponent link rather than a knot.

By Ng2, Prop. 4.4], Lemma 3.2 and Lemma 4.7, we have

$$
\begin{aligned}
\Phi_{\sigma_{k} B}^{L}(\Theta) & =\Phi_{B}^{L}\left(\Delta\left(v_{k}\right) \cdot \Theta \cdot \Delta\left(v_{k}\right)^{-1}\right) \cdot \Phi_{\sigma_{k}}^{L}(\Theta) \\
& =\Delta\left(s(B) v_{k}\right) \cdot \Phi_{B}^{L}(\Theta) \cdot \Delta\left(v_{k}\right)^{-1} \\
& =\Delta\left(u_{\sigma_{k} B}\right) \cdot \Delta\left(u_{B}\right)^{-1} \cdot \Phi_{B}^{L}(\Theta) \cdot\left(\left.\operatorname{Bur}_{\sigma_{k}}\right|_{t=-1 / \mu}\right) .
\end{aligned}
$$

Hence the formula holds for $B$ if and only if it holds for $\sigma_{k} B$. Since it certainly holds when $B$ is the trivial braid, the formula holds for general $B$ by induction.

Lemma 4.9. The distinguished augmentation is in fact an augmentation for the framed knot DGA of $B$, once we set $\lambda=\mu^{-w(B)}$. 
Proof. Define $\Theta_{B}=\Delta\left(v_{B}\right) \cdot \Theta \cdot \Delta\left(v_{B}\right)^{-1}$. We want to show that the map $A \mapsto \Theta_{B}$ sends the differentials of the degree 1 generators of the framed knot DGA to 0. By Lemmas 3.2 and 4.8, we have

$\Phi_{B}^{L}\left(\Theta_{B}\right)=\Delta\left(s(B) v_{B}\right) \cdot \Phi_{B}^{L}(\Theta) \cdot \Delta\left(v_{B}\right)^{-1}=\Lambda_{B}^{-1} \cdot \Delta\left(v_{B}\right) \cdot\left(\left.\operatorname{Bur}_{\hat{B}}\right|_{t=-1 / \mu}\right) \cdot \Delta\left(v_{B}\right)^{-1}$

and thus

$$
\left(1-\Lambda_{B} \cdot \Phi_{B}^{L}\left(\Theta_{B}\right)\right) \cdot \Theta_{B}=\Delta\left(v_{B}\right) \cdot\left(1-\left.\operatorname{Bur}_{\hat{B}}\right|_{t=-1 / \mu}\right) \cdot \Theta \cdot \Delta\left(v_{B}\right)^{-1}=0
$$

similarly $\Theta_{B} \cdot\left(1-\Phi_{B}^{R}\left(\Theta_{B}\right) \cdot \Lambda_{B}^{-1}\right)=0$.

Proof of Proposition 4.4 Let $B$ be a braid whose closure is $K$, and consider the linearization of the modified framed knot DGA of $B$, as defined in Section [3.1. By the calculation from the proof of Lemma 4.9, we have

$\partial_{\epsilon}^{\operatorname{lin}} F=B-C-\Lambda_{B} \cdot \Phi_{B}^{L}\left(\Theta_{B}\right) \cdot D=B-C-\Delta\left(v_{B}\right) \cdot\left(\left.\operatorname{Bur}_{\hat{B}}\right|_{t=-1 / \mu}\right) \cdot \Delta\left(v_{B}\right)^{-1} \cdot D$

and similarly $\partial_{\epsilon}^{\operatorname{lin}} E=B-D-C \cdot \Delta\left(v_{B}\right) \cdot\left(\left.\operatorname{Bur}_{\hat{B}}\right|_{t=-\mu}\right) \cdot \Delta\left(v_{B}\right)^{-1}$. If we change variables by replacing $B$ by $\Delta\left(v_{B}\right) \cdot B \cdot \Delta\left(v_{B}\right)^{-1}$ and similarly for $C, D, E, F$, then the differential becomes $\partial_{\epsilon}^{\operatorname{lin}} E=B-D-C \cdot\left(\left.\operatorname{Bur}_{\hat{B}}\right|_{t=-1 / \mu}\right)$, $\partial_{\epsilon}^{\operatorname{lin}} F=B-C-\left(\left.\operatorname{Bur}_{\hat{B}}\right|_{t=-\mu}\right) \cdot D$.

The matrices $\left.\operatorname{Bur}_{\hat{B}}\right|_{t=-1 / \mu}$ and $\left.\operatorname{Bur}_{\hat{B}}\right|_{t=-\mu}$ both give presentations over $\mathbb{Q}\left[t^{ \pm 1}\right]$ for the Alexander invariant of $K$, or, more precisely, for the direct sum of the Alexander polynomial and a free summand $\mathbb{Q}\left[t^{ \pm 1}\right]$, once we identify $t$ with $-\mu$. (Note that the Alexander invariant is unchanged if we invert t.) It is now straightforward to deduce the proposition; just use the method of the proof of $\mathrm{Ng} 2$, Prop. 7.8], working over the ring $\mathbb{Q}\left[\mu^{ \pm 1}\right]$ rather than $\mathbb{Z}$.

\section{InVARIANTS FROM Augmentation}

In this section, we focus on applications of framed knot contact homology. The essential difficulty is that it is a nontrivial task to determine when two DGAs over $\mathbb{Z}\left[\lambda^{ \pm 1}, \mu^{ \pm 1}\right]$ are equivalent. To obtain some computable invariants, we consider augmentations of the DGA. These give rise to a large family of readily calculable numerical invariants of knots (Section 5.1), as well as a 2 -variable polynomial with very strong ties to the $A$-polynomial (Section [5.2).

5.1. Augmentation numbers. Let $\mathbf{k}$ be a field, and $\mathbf{k}^{*}=\mathbf{k} \backslash\{0\}$. Any pair $\left(\lambda_{0}, \mu_{0}\right) \in\left(\mathbf{k}^{*}\right)^{2}$ gives rise to a map $\mathbb{Z}\left[\lambda^{ \pm 1}, \mu^{ \pm 1}\right] \otimes \mathbf{k} \rightarrow \mathbf{k}$ by sending $\lambda$ to $\lambda_{0}$ and $\mu$ to $\mu_{0}$. If $(\mathcal{A}, \partial)$ is a DGA over $\mathbb{Z}\left[\lambda^{ \pm 1}, \mu^{ \pm 1}\right]$, then $\left(\lambda_{0}, \mu_{0}\right)$ allows us to project $\mathcal{A} \otimes \mathbf{k}$, which is an algebra over $\mathbf{k}\left[\lambda^{ \pm 1}, \mu^{ \pm 1}\right]$, to an algebra over $\mathbf{k}$, which we write as $\left.\mathcal{A}\right|_{\left(\lambda_{0}, \mu_{0}\right)}$. 

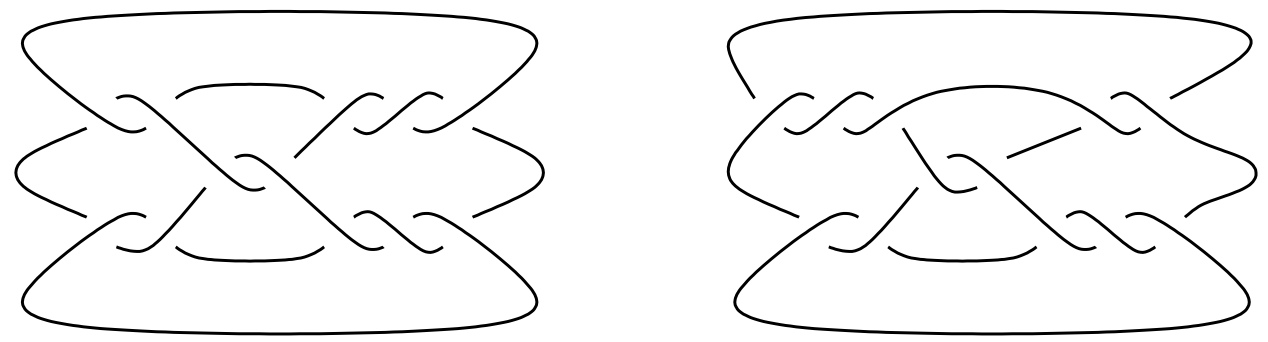

Figure 6. The Kinoshita-Terasaka knot (left) and Conway mutant (right).

Definition 5.1. An augmentation of a $\operatorname{DGA}(\mathcal{A}, \partial)$ over a field $\mathbf{k}$ is an algebra map $\varepsilon: \mathcal{A} \rightarrow \mathbf{k}$ such that $\varepsilon(1)=1, \varepsilon(a)=0$ if $a \in \mathcal{A}$ has pure nonzero degree, and $\varepsilon \circ \partial=0$.

If $\mathbf{k}$ is finite and $\mathcal{A}$ is finitely generated as an algebra, then there are only finitely many possible augmentations.

Definition 5.2. Let $K$ be a knot with framed DGA $(\mathcal{A}, \partial)$, let $\mathbf{k}$ be a finite field, and let $\left(\lambda_{0}, \mu_{0}\right) \in\left(\mathbf{k}^{*}\right)^{2}$. The augmentation number $\operatorname{Aug}\left(K, \mathbf{k}, \lambda_{0}, \mu_{0}\right)$ is the number of augmentations of $\left.\mathcal{A}\right|_{\left(\lambda_{0}, \mu_{0}\right)}$ over $\mathbf{k}$.

Since a knot has many different framed DGAs depending on its braid representative, Definition 5.2 technically requires proof of invariance; however, since framed knot DGAs have generators only in nonnegative degree, it is easy to see that an augmentation of $\left.\mathcal{A}\right|_{\left(\lambda_{0}, \mu_{0}\right)}$ is the same as an algebra $\left.\operatorname{map}\left(H C_{0}(K) \otimes \mathbf{k}\right)\right|_{\lambda=\lambda_{0}, \mu=\mu_{0}} \rightarrow \mathbf{k}$, and so augmentation numbers are welldefined.

From Section 2.4 if the bridge number of a knot $K$ is $m$, then there is a way to write $H C_{0}(K)$ as an algebra with $m(m-1)$ generators and $2 m^{2}$ relations. Calculating an augmentation number $\operatorname{Aug}\left(K, \mathbf{k}, \lambda_{0}, \mu_{0}\right)$ then involves counting how many of the possible $|\mathbf{k}|^{m(m-1)}$ maps of the generators to $\mathbf{k}$ satisfy the relations; this can be readily done by computer when $|\mathbf{k}|$ is small enough.

In particular, if $p$ is a prime, the finite field $\mathbb{Z}_{p}$ gives rise to $(p-1)^{2}$ augmentation numbers of a knot, corresponding to the different choices of $\left(\lambda_{0}, \mu_{0}\right)$, each of which is a knot invariant. It can be calculated without too much effort that nearly any pair of nonisotopic knots with 8 or fewer crossings can be distinguished by one of the 69 total augmentation numbers for $\mathbb{Z}_{p}$ with $p=2,3,5,7$. The only pairs not so distinguished are the knot $8_{15}$ and its mirror, and the connect sum $3_{1} \# 5_{1}$ and its mirror; each of these pairs share the same augmentation numbers over fields up to $\mathbb{Z}_{7}$ but can be distinguished using augmentation numbers over $\mathbb{Z}_{11}$.

As an application of augmentation numbers, we can calculate that the Kinoshita-Terasaka knot and its Conway mutant, as shown in Figure [6 do not have all the same augmentation numbers: $\operatorname{Aug}\left(\mathrm{KT}, \mathbb{Z}_{7}, 3,5\right)=2$ while 
$\operatorname{Aug}\left(\right.$ Conway, $\left.\mathbb{Z}_{7}, 3,5\right)=1$. The author's web page includes a Mathematica program to calculate augmentation numbers.

Proposition 5.3. The Kinoshita-Terasaka knot and its Conway mutant have nonisomorphic cord algebras (and thus inequivalent framed knot DGAs).

We conclude that augmentation numbers are a rather effective tool to distinguish between knots. It is not known to the author whether there are two nonisotopic knots which share the same augmentation numbers for all fields $\mathbb{Z}_{p}$.

5.2. The augmentation polynomial. Here we consider augmentations, as in the previous section, but now over the field $\mathbb{C}$. If we fix $\left(\lambda_{0}, \mu_{0}\right) \in\left(\mathbb{C}^{*}\right)^{2}$ and a knot $K$, then we obtain a $\operatorname{DGA}\left(\left.\mathcal{A}\right|_{\left(\lambda_{0}, \mu_{0}\right)}, \partial\right)$ over $\mathbb{C}$, and we can ask whether this DGA has an augmentation, i.e., whether there is an algebra $\left.\operatorname{map}\left(H C_{0}(K) \otimes \mathbb{C}\right)\right|_{\lambda=\lambda_{0}, \mu=\mu_{0}} \rightarrow \mathbb{C}$. Since $H C_{0}(K)$ is finitely generated and finitely presented, this is just a question in elimination theory, and the (closure of the) locus of $\left(\lambda_{0}, \mu_{0}\right)$ for which the DGA has an augmentation is an algebraic set.

Definition 5.4. Let $K$ be a knot, with framed knot DGA $(\mathcal{A}, \partial)$. The set $\left\{\left(\lambda_{0}, \mu_{0}\right) \in\left(\mathbb{C}^{*}\right)^{2}:\left(\left.\mathcal{A}\right|_{\left(\lambda_{0}, \mu_{0}\right)}, \partial\right)\right.$ has an augmentation $\}$ is called the augmentation variety of $K$. If the augmentation variety is not 2-dimensional (and thus a Zariski open subset of $\left(\mathbb{C}^{*}\right)^{2}$ ), then the union of its 1-dimensional components is the zero set of the augmentation polynomial $\tilde{A}_{K}(\lambda, \mu) \in$ $\mathbb{C}[\lambda, \mu]$, which is unique up to constant multiplication once we specify that it has no repeated factors and is not divisible by $\lambda$ or $\mu$.

The author does not know of any examples for which the augmentation variety has any components which are not 1-dimensional.

Conjecture 5.5. The augmentation polynomial is defined for all knots in $S^{3}$.

Proposition 5.6. The augmentation polynomial $\tilde{A}_{K}(\lambda, \mu)$ is always divisible by $(\lambda-1)(\mu+1)$.

Proof. If $\lambda_{0}=1$, then there is a map from $\left.H C_{0}(K)\right|_{\left(1, \mu_{0}\right)}$ to $\mathbb{C}$ sending all cords to $1+\mu$; if $\mu_{0}=-1$, then there is a trivial map from $\left.H C_{0}(K)\right|_{\left(\lambda_{0},-1\right)}$ to $\mathbb{C}$ sending all cords to 0 .

We computed in Section 2.1 that the cord algebra for the unknot is $\mathbb{Z}\left[\lambda^{ \pm 1}, \mu^{ \pm 1}\right] /((\lambda-1)(\mu+1))$; hence the unknot has augmentation polynomial

$$
\tilde{A}_{0}(\lambda, \mu)=(\lambda-1)(\mu+1) .
$$

A slightly more interesting case is the right hand trefoil, for which we computed in Section 2.2 that the cord algebra is $\left(\mathbb{Z}\left[\lambda^{ \pm 1}, \mu^{ \pm 1}\right]\right)[x] /\left(x^{2}-x-\right.$ $\left.\lambda \mu^{2}-\lambda \mu, x^{2}-\lambda \mu x-\lambda-\lambda \mu\right)$. For this to have a map to $\mathbb{C}$, we need the two polynomials generating the ideal to have a common root. Their resultant 
is $\lambda(\lambda-1)(\mu+1)\left(1-\lambda \mu^{3}\right)$, and so the right hand trefoil has augmentation polynomial

$$
\tilde{A}_{3_{1}}(\lambda, \mu)=(\lambda-1)(\mu+1)\left(1-\lambda \mu^{3}\right) .
$$

We will give some more computations of augmentation polynomials shortly, but first we establish a couple more properties of augmentation polynomials. For Laurent polynomials in $\mathbb{Z}\left[\lambda^{ \pm 1}, \mu^{ \pm 1}\right]$, let $\doteq$ denote equality up to multiplication by a unit in $\mathbb{Z}\left[\lambda^{ \pm 1}, \mu^{ \pm 1}\right]$.

Proposition 5.7. The augmentation polynomial is independent of the orientation of the knot. If $K$ is a knot with mirror $\bar{K}$, then

$$
\tilde{A}_{K}(\lambda, \mu) \doteq \tilde{A}_{K}\left(\lambda^{-1}, \mu^{-1}\right) \quad \text { and } \quad \tilde{A}_{\bar{K}}(\lambda, \mu) \doteq \tilde{A}_{K}\left(\lambda, \mu^{-1}\right) .
$$

Proof. Follows immediately from Proposition 4.1. note that the augmentation polynomial depends only on the commutative framed cord algebra, rather than the noncommutative version.

Proposition 5.8. The augmentation variety $V_{K_{1} \# K_{2}}$ for a connect sum $K_{1} \# K_{2}$ is related to the augmentation varieties $V_{K_{1}}, V_{K_{2}}$ for $K_{1}, K_{2}$ as follows:

$$
V_{K_{1} \# K_{2}}=\left\{\left(\lambda_{1} \lambda_{2}, \mu\right) \in\left(\mathbb{C}^{*}\right)^{2} \mid\left(\lambda_{1}, \mu\right) \in V_{K_{1}} \text { and }\left(\lambda_{2}, \mu\right) \in V_{K_{2}}\right\} .
$$

This formula defines $\tilde{A}_{K_{1} \# K_{2}}$ in terms of $\tilde{A}_{K_{1}}$ and $\tilde{A}_{K_{2}}$. In particular, both $\tilde{A}_{K_{1}}$ and $\tilde{A}_{K_{2}}$ divide $\tilde{A}_{K_{1} \# K_{2}}$.

Proof. Since $\{\mu=-1\}$ is a component of all augmentation varieties, it suffices to prove the equality in the statement in the complement of this component.

By Van Kampen's Theorem, $\pi_{1}\left(S^{3} \backslash\left(K_{1} \# K_{2}\right)\right)$ is the free product of $\pi_{1}\left(S^{3} \backslash K_{1}\right)$ and $\pi_{1}\left(S^{3} \backslash K_{2}\right)$, modulo the identification of the two meridians. Thus there are elements $l_{1}, l_{2}, m \in \pi_{1}\left(S^{3} \backslash\left(K_{1} \# K_{2}\right)\right)$ such that $l_{1} l_{2}, m$ are the longitude and meridian classes of $K_{1} \# K_{2}$, and $l_{i}, m$ project to the longitude and meridian classes in $\pi_{1}\left(S^{3} \backslash K_{i}\right)$ for $i=1,2$.

A map $p:\left.H C_{0}\left(K_{1} \# K_{2}\right)\right|_{\left(\lambda_{0}, \mu_{0}\right)} \rightarrow \mathbb{C}$ with $\mu_{0} \neq-1$ sends $\left[l_{1}\right],\left[l_{2}\right]$ to complex numbers which we write as $\lambda_{0,1}\left(1+\mu_{0}\right), \lambda_{0,2}\left(1+\mu_{0}\right)$. We claim that $p$ induces maps from $\left.H C_{0}\left(K_{i}\right)\right|_{\left(\lambda_{0, i}, \mu_{0}\right)}$ to $\mathbb{C}$. The only relation for $\left.H C_{0}\left(K_{i}\right)\right|_{\left(\lambda_{0, i}, \mu_{0}\right)}$ from Definition 2.2 that is not trivially preserved by $p$ is $\left[\gamma l_{i}\right]=\left[l_{i} \gamma\right]=\lambda_{0, i}[\gamma]$. But in $\left.H C_{0}\left(K_{1} \# K_{2}\right)\right|_{\left(\lambda_{0}, \mu_{0}\right)}$, we have

$$
[\gamma]\left[l_{i}\right]=\left[\gamma l_{i}\right]+\left[\gamma m l_{i}\right]=\left[\gamma l_{i}\right]+\left[\gamma l_{i} m\right]=\left(1+\mu_{0}\right)\left[\gamma l_{i}\right],
$$

and so $p\left(\left[\gamma l_{i}\right]\right)=p\left([\gamma]\left[l_{i}\right]\right) /\left(1+\mu_{0}\right)=\lambda_{0, i} p([\gamma])$. Similarly $p\left(\left[l_{i} \gamma\right]\right)=\lambda_{0, i} p([\gamma])$, and so $p$ does indeed restrict to maps from $\left.H C_{0}\left(K_{i}\right)\right|_{\left(\lambda_{0, i}, \mu_{0}\right)}$ to $\mathbb{C}$. In addition, we have

$$
\lambda_{0}\left(1+\mu_{0}\right)=p([l])=p\left(\left[l_{1} l_{2}\right]\right)=\lambda_{0,1} p\left(\left[l_{2}\right]\right)=\lambda_{0,1} \lambda_{0,2}\left(1+\mu_{0}\right) .
$$

We conclude that if $\left(\lambda_{0}, \mu_{0}\right) \in V_{K_{1} \# K_{2}}$, then there exist $\lambda_{0,1}, \lambda_{0,2}$ with $\lambda_{0,1} \lambda_{0,2}=\lambda_{0}$ such that $\left(\lambda_{0, i}, \mu_{0}\right) \in V_{K_{i}}$ for $i=1,2$. 
Conversely, suppose that we are given maps $p_{i}:\left.H C_{0}\left(K_{i}\right)\right|_{\left(\lambda_{0, i}, \mu_{0}\right)} \rightarrow \mathbb{C}$. We construct a map $p:\left.H C_{0}\left(K_{1} \# K_{2}\right)\right|_{\left(\lambda_{0,1} \lambda_{0,2}, \mu_{0}\right)} \rightarrow \mathbb{C}$ as follows: if $\gamma_{j, i} \in$ $\pi_{1}\left(S^{3} \backslash K_{i}\right)$ for $i=1,2$ and $1 \leq j \leq k$, then

$$
p\left(\left[\gamma_{1,1} \gamma_{1,2} \cdots \gamma_{k, 1} \gamma_{k, 2}\right]\right)=\frac{p_{1}\left(\left[\gamma_{1,1}\right]\right) p_{2}\left(\left[\gamma_{1,2}\right]\right) \cdots p_{1}\left(\left[\gamma_{k, 1}\right]\right) p_{2}\left(\left[\gamma_{k, 2}\right]\right)}{\left(1+\mu_{0}\right)^{k-1}} .
$$

Since any element of $\pi_{1}\left(S^{3} \backslash\left(K_{1} \# K_{2}\right)\right)$ can be written in the form $\gamma_{1,1} \gamma_{1,2} \cdots \gamma_{k, 1} \gamma_{k, 2}$, this defines $p$ on $\left.H C_{0}\left(K_{1} \# K_{2}\right)\right|_{\left(\lambda_{0,1} \lambda_{0,2}, \mu_{0}\right)}$. It is straightforward to check that $p$ preserves the relations defining $\left.H C_{0}\left(K_{1} \# K_{2}\right)\right|_{\left(\lambda_{0,1} \lambda_{0,2}, \mu_{0}\right)}$ and is hence well-defined.

To illustrate Proposition [5.8 we can calculate that the augmentation polynomial for the connect sum of two right hand trefoils is

$$
\tilde{A}_{3_{1} \# 3_{1}}(\lambda, \mu)=(\lambda-1)(\mu+1)\left(1-\lambda \mu^{3}\right)\left(1-\lambda \mu^{6}\right),
$$

while the augmentation polynomial for the connect sum of a left hand trefoil and a right hand trefoil is

$$
\tilde{A}_{3_{1} \# \overline{3_{1}}}(\lambda, \mu)=(\lambda-1)(\mu+1)\left(1-\lambda \mu^{3}\right)\left(\lambda-\mu^{3}\right) .
$$

5.3. The augmentation polynomial and the $A$-polynomial. The augmentation polynomial is very closely related to the $A$-polynomial introduced by Cooper et al. in CCGLS, which we briefly review here. Let $K \in S^{3}$ be a knot, and let $l, m$ denote the longitude and meridian classes in $\pi_{1}\left(S^{3} \backslash K\right)$, as usual. Consider a representation $\rho: \pi_{1}\left(S^{3} \backslash K\right) \rightarrow S L_{2} \mathbb{C}$. Since $l, m$ commute, $\rho$ is conjugate to a representation in which $\rho(l)$ and $\rho(m)$ are upper triangular, and so we restrict our attention to representations $\rho$ with this property. There is a map from $S L_{2} \mathbb{C}$-representations of $\pi_{1}\left(S^{3} \backslash K\right)$ to $\left(\mathbb{C}^{*}\right)^{2}$ sending $\rho$ to $\left((\rho(l))_{11},(\rho(m))_{11}\right)$, where $M_{11}$ denotes the top left entry of $M$, and the 1-dimensional part of the image of this map forms a variety, which is the zero set of the $A$-polynomial $A_{K} \in \mathbb{C}[\lambda, \mu]$. (Note that we are thus assuming the convention that $\lambda-1$ always divides $A_{K}$.)

The following result shows that the $A$-polynomial is subsumed in the $\tilde{A}$ polynomial. Note that the $A$-polynomial involves only even powers of $\mu$ CCGLS, Prop. 2.9].

Proposition 5.9. Let $A_{K}(\lambda, \mu), \tilde{A}_{K}(\lambda, \mu)$ denote the A-polynomial and augmentation polynomial of a knot $K$. Then

$$
\left(1-\mu^{2}\right) A_{K}(\lambda, \mu) \mid \tilde{A}_{K}\left(\lambda,-\mu^{2}\right) .
$$

Proof. From [CCGLS, §2.8], $\mu \pm 1$ does not divide the $A$-polynomial. On the other hand, by Proposition [5.6, $\tilde{A}_{K}\left(\lambda,-\mu^{2}\right)$ is divisible by $1-\mu^{2}$. It thus suffices to prove that $A_{K}(\lambda, \mu)$ divides $\tilde{A}_{K}\left(\lambda,-\mu^{2}\right)$.

Suppose that we have an $S L_{2} \mathbb{C}$-representation $\rho$ of $\pi_{1}\left(S^{3} \backslash K\right)$ which maps to $\left(\lambda_{0}, \mu_{0}\right)$ in $\left(\mathbb{C}^{*}\right)^{2}$. We wish to show that there is a map from the cord 
algebra of $K$ with $(\lambda, \mu)=\left(\lambda_{0},-\mu_{0}^{2}\right)$ to $\mathbb{C}$. Since $\mu+1$ is a factor of $\tilde{A}_{K}(\lambda, \mu)$, we may assume that $\mu_{0} \neq \pm 1$. Then we can conjugate $\rho$ so that it becomes diagonal on the peripheral subgroup:

$$
\rho(l)=\left(\begin{array}{cc}
\lambda_{0} & 0 \\
0 & \lambda_{0}^{-1}
\end{array}\right) \quad \text { and } \quad \rho(m)=\left(\begin{array}{cc}
\mu_{0} & 0 \\
0 & \mu_{0}^{-1}
\end{array}\right) .
$$

We use the formulation of the cord algebra from Definition 2.2, with $\lambda$ replaced by $\lambda_{0}$ and $\mu$ replaced by $-\mu_{0}^{2}$; we can change variables by replacing each generator $[\gamma]$ by $\left(1-\mu_{0}^{2}\right)\left(-\mu_{0}\right)^{1 \mathrm{k}(\gamma, K)}[\gamma]$ to obtain an algebra with defining relations

(1) $[e]=1$;

(2) $[\gamma m]=[m \gamma]=\mu_{0}[\gamma]$ and $[\gamma l]=[l \gamma]=\lambda_{0}[\gamma]$;

(3) $\left[\gamma_{1} \gamma_{2}\right]-\mu_{0}\left[\gamma_{1} m \gamma_{2}\right]=\left(1-\mu_{0}^{2}\right)\left[\gamma_{1}\right]\left[\gamma_{2}\right]$.

Now if we send each generator $[\gamma]$ to the top left entry of $\rho(\gamma)$, it is easy to check that the above relations are preserved, and so we obtain a map from the cord algebra to $\mathbb{C}$ with $(\lambda, \mu)=\left(\lambda_{0},-\mu_{0}^{2}\right)$, as desired.

Proposition 5.9 has some immediate consequences. The first uses a result of Dunfield and Garoufalidis DG. which is based on work of Kronheimer and Mrowka [KM] on knot surgery and $S U_{2}$ representations.

Proposition 5.10. The cord algebra distinguishes the unknot from any other knot in $S^{3}$.

Proof. We have seen that the cord algebra for the unknot is $\mathbb{Z}\left[\lambda^{ \pm 1}, \mu^{ \pm 1}\right] /((\lambda-$ $1)(\mu+1))$, and so the augmentation variety for the unknot is the union of the lines $\lambda=1$ and $\mu=-1$. On the other hand, the main result of [DG] states that any nontrivial knot in $S^{3}$ has nontrivial $A$-polynomial (i.e., not equal to $\lambda-1$ ). Hence the augmentation variety for a nontrivial knot either is 2-dimensional or contains a nontrivial 1-dimensional component; in either case, it strictly contains the augmentation variety for the unknot.

The next consequence of Proposition 5.9 relates the augmentation variety to the Alexander polynomial. By analogy with [CCGLS], say that a point $\left(\lambda_{0}, \mu_{0}\right)$ in the augmentation variety of $K$ is reducible if $\lambda_{0}=1$ and the corresponding map from the cord algebra (from Definition 2.4) to $\mathbb{C}$ sends every cord to $1+\mu_{0}$. (This is slightly bad notation: we mean a point which is on a component of the augmentation variety different from $\lambda-1$, with the corresponding map from the cord algebra to $\mathbb{C}$.)

Proposition 5.11. If $-\mu_{0}$ is a root of the Alexander polynomial $\Delta_{K}(t)$, then $\left(1, \mu_{0}\right)$ is a reducible point on a nontrivial component of the augmentation variety of $K$.

Proof. This follows directly from [CCGLS, Prop. 6.2].

We now examine the difference between the augmentation polynomial and the $A$-polynomial for some specific knots. It seems that the two polynomials 
essentially coincide for 2-bridge knots, but as of this writing, a proof has not been completed, and so the following "result" is presented as a conjecture.

Conjecture 5.12. If $K$ is a 2-bridge knot, then

$$
\left(1-\mu^{2}\right) A_{K}(\lambda, \mu) \doteq \tilde{A}_{K}\left(\lambda,-\mu^{2}\right) .
$$

On the other hand, there are knots for which $\tilde{A}_{K}\left(\lambda,-\mu^{2}\right)$ contains nontrivial factors not present in $A_{K}(\lambda, \mu)$. Define the polynomial $B_{K}(\lambda, \mu)$, defined up to multiplication by constants, to be the product of the factors of $\tilde{A}_{K}(\lambda, \mu)$, besides $\mu+1$, which do not have a corresponding factor in $A_{K}(\lambda, \mu)$; that is,

$$
B_{K}\left(\lambda,-\mu^{2}\right)=\frac{\tilde{A}_{K}\left(\lambda,-\mu^{2}\right)}{\left(1-\mu^{2}\right) A_{K}(\lambda, \mu)} .
$$

Conjecture 5.12 states that $B_{K}=1$ when $K$ is a 2-bridge knot.

Suppose that $K$ is the torus knot $T(3,4)$. The group $\pi_{1}\left(S^{3} \backslash T(3,4)\right)$ has presentation $\left\langle x, y: x^{3}=y^{4}\right\rangle$, with peripheral classes $m=x y^{-1}$ and $l=x^{3} m^{-12}$. A quick calculation then shows that

$$
A_{T(3,4)}(\lambda, \mu)=(\lambda-1)\left(1+\lambda \mu^{12}\right)\left(1-\lambda \mu^{12}\right) \text {. }
$$

On the other hand, we can define the cord algebra for $T(3,4)$ from Definition [2.6] using the braid $\left(\sigma_{1} \sigma_{2}\right)^{-4} \in B_{3}$ which closes to $T(3,4)$. It is straightforward to check that if we set $\lambda=\mu^{-8}$ and $a_{12}=a_{21}=a_{13}=a_{31}=$ $a_{23}=a_{32}=-1$, then the relations defining the cord algebra for $T(3,4)$ vanish, and so $1-\lambda \mu^{8}$ is a factor of $\tilde{A}_{T(3,4)}$. In fact, a slightly more involved calculation using resultants demonstrates that

$$
B_{T(3,4)}(\lambda, \mu)=1-\lambda \mu^{8} \neq 1 .
$$

Using Mathematica, one can compute the augmentation polynomials for many other knots. In particular, for the non-two-bridge knots whose $A$ polynomials are computed in CCGLS, we have

$$
\begin{aligned}
B_{8_{5}}(\lambda, \mu) & =1-\lambda \mu^{4} ; \\
B_{8_{20}}(\lambda, \mu) & =1-\lambda \mu^{2} ; \\
B_{P(-2,3,7)}(\lambda, \mu) & =1-\lambda \mu^{12} .
\end{aligned}
$$

The author does not know whether there is any geometric significance to the $B$-polynomial, as there is for the $A$-polynomial. 


\section{REFERENCES}

[Che] Yu. Chekanov, Differential algebra of Legendrian links, Invent. Math. 150 (2002), no. 3, 441-483.

[CCGLS] D. Cooper, M. Culler, H. Gillet, D. D. Long, and P. B. Shalen, Plane curves associated to character varieties of 3-manifolds, Invent. Math. 118 (1994), 4784 .

[DG] N. Dunfield and S. Garoufalidis, Non-triviality of the $A$-polynomial for knots in $S^{3}$, preprint, arXiv:math.GT/0405353.

[EES] T. Ekholm, J. Etnyre, and M. Sullivan, Legendrian submanifolds in $\mathbb{R}^{2 n+1}$ and contact homology, arXiv:math.SG/0210124.

[Eli] Ya. Eliashberg, Invariants in contact topology, Doc. Math. 1998, Extra Vol. II, 327-338 (electronic).

[EGH] Ya. Eliashberg, A. Givental, and H. Hofer, Introduction to symplectic field theory, GAFA 2000 (Tel Aviv, 1999), Geom. Funct. Anal. 2000, Special Volume, Part II, 560-673; also available as arXiv:math.SG/0010059.

[ENS] J. Etnyre, L. Ng, and J. Sabloff, Invariants of Legendrian knots and coherent orientations, J. Symplectic Geom. 1 (2002), no. 2, 321-367; also available as arXiv:math.SG/0101145.

[KM] P. Kronheimer and T. Mrowka, Dehn surgery, the fundamental group and $S U(2)$, preprint, arXiv:math.GT/0312322.

[Ng1] L. Ng, Computable Legendrian invariants, Topology 42 (2003), no. 1, 55-82; also available as arXiv:math.GT/0011265.

[Ng2] L. Ng, Knot and braid invariants from contact homology I, preprint, arXiv: math.GT/0302099 v3.

[Ng3] L. Ng, Knot and braid invariants from contact homology II, preprint, arXiv : math.GT/0303343 v2.

[Rol] D. Rolfsen, Knots and Links (Publish or Perish Press, Berkeley, 1976).

Department of Mathematics, Stanford University, Stanford, CA 94305

E-mail address: lng@math.stanford.edu

$U R L:$ http://alum.mit.edu/www/ng 